\title{
A Model of Socially Sustained Self-Tracking for Food and Diet
}

\author{
XI LU, University of California, Irvine, USA \\ YUNAN CHEN, University of California, Irvine, USA \\ DANIEL A. EPSTEIN, University of California, Irvine, USA
}

\begin{abstract}
Studies of personal informatics systems primarily examine people's use or non-use, but people often leverage other technology towards their long-term behavior change processes such as social platforms. We explore how tracking technologies and social platforms together help people build healthy eating behaviors by interviewing 18 people who use Chinese food journaling apps. We contribute a Model of Socially Sustained Self-Tracking in personal informatics, building on the past model of Personal Informatics and the learning components of Social Cognitive Theory. The model illustrates how people get advice from social platforms on when and how to track, transfer data to and apply knowledge from social platforms, evolve to use social platforms after tracking, and occasionally resume using tracking tools. Observational learning and enactive learning are central to these processes, with social technologies helping people to gain deeper and more reliable domain knowledge. We discuss how lapsing and abandoning of tracking can be viewed as evolving to social platforms, offering recommendations for how technology can better facilitate this evolution.
\end{abstract}

CCS Concepts: • Human-centered computing $\rightarrow$ Human-computer interaction (HCI); HCI theory, concepts and models; User Studies; Social media

KEYWORDS: Personal Informatics; Self-Tracking; Social Media; Social Platforms; Food; Model

\section{ACM Reference format:}

Xi Lu, Yunan Chen, Daniel A. Epstein. 2021. A Model of Socially Sustained Self-Tracking for Food and Diet. In PACM on Human Computer Interaction, Vol. 5, CSCW2, Article 451, October 2021. ACM, New York, NY, USA. 32 pages. https://doi.org/10.1145/3479595

\section{INTRODUCTION}

People have varied motivations for using technology to track their fitness, eating, finances, or other habits. Many of these goals focus on self-improvement, such as changing behavior or becoming more aware of activities [28,47]. Much of the HCI and CSCW community's knowledge around how people use personal tracking tools has been described in conceptual models of personal informatics, such as Li et al.'s Stage-Based Model [47], Epstein et al.'s Lived Informatics Model [28], and Niess \& Woźniak's Tracker Goal Evolution Model [62]. These models help surface different goals people have for self-tracking, how those goals evolve over time, and how people's use of technology changes over their tracking lifetime. Insights from these models have helped the

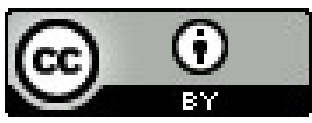

This work is licensed under a Creative Commons Attribution International 4.0 License.

(C) 2021 Copyright held by the owner/author(s).

2573-0142/2021/10-ART454. https://doi.org/10.1145/3479595 
community create new tools for helping people derive more value from the data they collect $[16,31,36]$ and better identify their goals [50], and have provided lenses for understanding people's experiences with tracking, such as around fertility [22] or in tracking as a family [56].

The self-improvement goals that people turn to personal tracking technology to help address or monitor are often complex and long-term processes, occurring over years and intersecting with everyday challenges [70]. Although tracking technology can contribute to long-term behavior change [38,58], today's models and empirical studies tend to mainly focus on people's use or nonuse of tracking tools, reporting on successful actions or changes resulting from tracking or reasons for abandonment. Though research has examined how people leverage tracking tools and additional systems to support reflection [9], more work is necessary to understand how people use other kinds of technology during or after tracking, when the benefits of tracking diminish, the burdens become too high, or other technologies better support people's evolving needs.

Compared to tracking technology, social technologies like video sites, Q\&A sites, and social networking sites serve different and often complimentary roles in supporting behavior change. People often turn to social technologies to share their progress, seek support, or elicit advice to sustain or inform behavior change [16,22,27]. Understanding how people's use of social technologies intersect with their use of tracking technologies towards long-term processes of initiating, building, and maintaining healthy habits can help us understand how different tools fit into people's use of technology for self-improvement or behavior change.

We specifically examine how people's use of food and diet tracking technology intersects with their use of social technology to establish healthy eating practices. Food journaling is a particularly popular tracking domain, with some food journaling apps being downloaded over 50 million times [29]. We investigate people's practices through interviews with 18 current and former users of Chinese food journaling apps. We use the enactive learning, learning from doing, and observational learning, learning via observation, of Social Cognitive Theory (SCT) $[5,44,64,86]$ to describe the learning behaviors that occur during and after tracking along people's long-term behavior change processes. Together with participants' other practices around self-tracking and social technology use for food and diet tracking, built on the Lived Informatics Model [28], we develop a new Model of Socially Sustained Self-Tracking.

Our findings suggest that people's food journaling practices are often intertwined with social platforms. People's decision to track and selection of tools are often influenced by content they encounter on social platforms, they then leverage social platforms to share their tracking data or progress. People's desire to learn knowledge crucial for self-improvement drives them to migrate between tracking and social technology when behavior change progresses as these two technologies enable different learning behaviors. Once they acquire sufficient understanding and knowledge from the enactive learning process provided by tracking tools, they often evolve to social platforms. There, they continue learning by finding and observing more reliable and more specialized domain knowledge that meet their specific goals. People may occasionally return to tracking when they need to learn nutrients of specific foods or to restart a new goal that food journaling apps can help. Individual use of tracking technology and collective use of social technology co-evolve to help people sustain long-term health behavior change.

This work makes three contributions to CSCW and personal informatics:

1. Empirical insights on how tracking and social technologies collectively support people's selfimprovement and behavior change needs. We highlight that people often seek out food journaling apps with a desire to learn best practices for healthy eating, extending current 
discussions that tracking primarily aims to support self-understanding. We also report on how people select and leverage social platforms to satisfy their social sharing needs both during and after self-tracking.

2. A theoretical Model of Socially Sustained Self-Tracking which articulates how people's use of food journaling technology intersect with social platforms. Our model incorporates enactive learning and observational learning from Social Cognitive Theory to describe how different technologies support people's learning needs. Our model describes how people get advice on when and how to track from others from social platforms, transfer data to social platforms to satisfy social needs and sustain tracking practices, learn basic self and domain knowledge from both the tracking tool itself and social platforms, evolve to use social platforms after tracking, and occasionally resume the use of tracking tools. Additionally, our data suggests that tracking technologies serve as a gateway to social technologies during people's overall behavior change process.

3. Design opportunities for food journaling apps and social platforms to better support people's learning and tracking needs. Tracking tools can better serve as a gateway to guide people towards learning appropriate healthy eating practices on external social platforms by arming people with necessary and reliable self and domain knowledge, such as combatting misinformation about best practices for healthy eating that pervade on social platforms. Social features inside tracking apps can be rethought to better support transition between tracking technology and social technology.

\section{BACKGROUND}

Personal informatics has proposed conceptual models of how people track, describing the challenges people face in achieving their varied goals. Food tracking presents further domainspecific challenges. Our model and findings draw on prior work on personal informatics models, food tracking, social tracking and sharing, and Social Cognitive Theory.

\subsection{Personal Informatics and Its Models}

People leverage personal informatics systems to collect personal data for self-reflection and learning self-knowledge [47]. Choe et al. studied the Quantified Self movement, realizing people are motivated to either improve themselves or discover new life experiences through self-tracking [15]. Rooksby et al. further proposed the concept of lived informatics that tracking needs to be situated in and understood through day to day activities [70].

Several models describe the tools people use for tracking and activities they undertake, such as Li et al. [47]'s Stage-Based Personal Informatics Model, Epstein et al.'s Lived Informatics Model [28], and Niess \& Woźniak's Tracker Goal Evolution Model [62]. Epstein et al.'s Lived Informatics Model, which we use as an analytic lens, describes people's use of self-trackers in everyday life [28]. The model describes people deciding and selecting tracking tools out of curiosity or deliberately depending on tracking goals, tracking and acting by simultaneously collecting, integrating, and reflecting on personal data, lapsing in tracking intentionally and accidentally, and resuming to either the same tracking tool or selecting a new tool.

Prior models primarily focus on use or non-use of self-tracking systems, exploring factors related to tracking success or failure. For example, Li et al. [47] described barriers that people often experience at different stages of tracking, and Epstein et al. [28] identified common reasons people abandon tracking. Clawson et al. [17] further identified abandonment reasons beyond devices' lack 
of useful utilities, finding that abandonment often occurs due to devices' inability to support individual's evolving needs. Prior studies also identified happy abandonment, when tracking is no longer needed as people reach their goals or learn enough [17,24]. Past work has also primarily regarded self-tracking as a self-contained activity. Although people might use multiple tools collaboratively to track [28,70], less is known about how tracking fits into broader ecosystems of technology use to support self-improvement.

Most people only track persistently for a few months at a time, often shorter depending on the burdens of the domain, which is often before long-term behavior change is achieved [58]. People often abandon tracking due to changes in their goals and life circumstances [17,24], dissatisfaction with their selected tracking tools [17,24,28,45], and the emotional cost of tracking and sharing [24]. People also abandon tracking after successfully achieving their tracking goals such as changing their habits or gaining enough knowledge [17,24]. Recent work has suggested using other forms of technology to support continuing people's behavior change after they abandon tracking. For example, Burgess et al. [11] proposed that technology ecosystems containing mobile apps, social media, and online communities can support people's self-management towards mental health. We explore where self-tracking systems might fit into broader technology ecosystems to support longterm and complex behavior change around healthy eating.

\subsection{Food Tracking in Daily Life For Self-Improvement}

Although food tracking is a popular practice in our daily lives, previous studies found generally low adherence and sustained use of food journaling apps [38]. The effort required to journal, particularly manual logging of food, becomes a major barrier for persistent use [21]. To maintain motivation, food journaling apps leverage many behavior change techniques, such as reminders, rewards, and social features [75]. Sociality is particularly important to people's tracking practices, and some people use typically-social platforms to journal and share their food. For example, Chung at el. examined why and how users appropriate Instagram to track and share food data, finding that people socially track not only to record, but also to seek social support [16].

Some food tracking systems also focus on providing people with information about healthy eating and their own habits to help with self-improvement. Most people journal food either to build a healthier diet or reach a more satisfying weight [21], which requires people to obtain knowledge about how to approach healthy eating to take appropriate actions [63]. Prior work has proposed that technology can provide professional dietary knowledge to raise their awareness of healthy eating [34], such as through counselors providing health and nutrition knowledge [83]. Besides domain knowledge, self-knowledge about eating habits can enable people to reflect on and make changes to their daily practices. Technology systems have used visualizations and summarizing collections of photographs to educate users about the relationship between daily diet and physical activities to help them reflect and make better health choices $[10,32]$. However, even with features deliberately designed to sustain food logging, many people only continue using food journaling apps a few months or less $[38,58]$. We therefore explore how tracking technology relates to other kinds of technology to support people towards self-improvement of food and diet.

\subsection{Social Aspects of Personal Tracking}

Social interaction can help sustain engagement in long-term tracking [25,31,65,77]. Often, social features of tracking tools aim to connect people with others, such as sharing data to facilitate conversation [52,53] or to raise awareness of the healthy habits that others are partaking in [7]. 
Some systems have also supported people in sharing accomplishments they have tracked [26]. Other designs have studied or supported instrumental sharing goals, such as enabling people to share their tracking process, collectively making sense of self-tracked data, or use their data to facilitate recommendations on what to do next [38]. Social features can also introduce comparison and competition through rankings, leaderboards, interpersonal competitions, and even viewing other users' shared logs and profiles [69].

Social technologies are systems primarily designed for supporting interpersonal communication including social networking sites (e.g., Facebook, Twitter, Instagram) or messaging channels (e.g., SMS, Email) [43]. Although social technology is not explicitly designed for personal tracking, people sometimes appropriate its features to support tracking needs even alongside other tracking tools [16]. Additionally, though many self-tracking tools have their own social features, they support exporting tracked data to traditionally social platforms for support [16,25], instrumental sharing [2,36,65], and comparison [2,25,36]. People's motivations for using dedicated social platforms are similar to people's use of tracking tools' incorporated social features. Chung et al. [16] suggest that tracking on social platforms can help people combine self-understanding with receiving and providing social support. Gui et al. [36] further report that people share data with pre-existing social networks, such as their friends and families, on private groups on messaging platforms. However, previous studies typically examine people's use of one social platform for social support around behavior change, such as Instagram [2,16], Twitter [27,65,78], Facebook [25,61], or WeChat [36]. Sleeper et al. [74] suggest that people tend to selectively choose with whom to share on social media, what channel to use, and whether to share on more than one channel. We examine people's holistic use of multiple social platforms to understand the respective roles of different platforms, how people migrate among or between platforms, and challenges that people face in using each.

\subsection{Social Cognitive Theory}

The Social Cognitive Theory (SCT) evolves from Bandura's social learning theory (SLT), arguing that people can learn new behaviors by proactively observing and imitating what others do, referred to as observational learning [6,35]. SCT also defines enactive learning, whereby people obtain knowledge by directly performing a behavior [5]. SCT further proposes that people's behaviors are informed by the combination of their own experiences, others' behaviors, and the situated social environment $[5,44,64,86]$. SCT has been widely used to understand and design interventions for health behavior change $[18,44,86]$ by uncovering and supporting how people initiate, perform, and maintain healthy behaviors.

According to Bandura, the process of observational learning includes four necessary components: attention, retention, production, and motivation [4,5,60,84]. Attention is what information people selectively pay attention to when observing others' behaviors, such as noticing others' healthy eating practices in a social setting. Retention is people's internal cognitive process for remembering a behavior they observed, such as remembering others' healthy eating practices after observing them. Production is whether people are able to perform the behavior they observed themselves by understanding its course of action, such as knowing how to conduct the same healthy eating practices they observed. Motivation is people's willingness to proactively perform a behavior.

Observing others' behaviors and learning how to act is not sufficient for positive behavior change. People may hold a negative attitude about whether they can succeed in performing similar 
behaviors. According to SCT, self-efficacy plays a central role in determining whether people are motivated to take action and how well they perform a behavior [3]. Self-efficacy represents people's beliefs in their ability to execute a certain behavior and sustain it when facing challenges $[5,44,86]$. One major source is the vicarious experience, meaning observing the success of others they see as models, especially people who are like themselves [18,44].

Some scholars in HCI and CSCW have leveraged SCT to design or evaluate interventions that facilitate behavior change. Several studies explored how people observationally learn from others on online platforms to acquire new knowledge. For example, Ammari et al. examined how fathers observe others' parenting decisions and outcomes on social media to avoid making similar mistakes [1]. Observational learning in health technology, such as mobile apps for tracking and sharing exercise and general health behaviors, can increase people's awareness of the decisions that others make $[7,82]$. In designing StoryMap, Saksono et al. leveraged the observational learning component of SCT, finding that it can enhance key attitudes, such as self-efficacy, to promote physical activity [71]. Enactive learning is also used in health interventions to increase people's selfefficacy. Peng [67] leveraged the enactive learning in a game to promote young people's perceptions of healthy diet, creating a virtual space for managing food choices. Extending on these designs, we specifically examine how tracking and social technology each support the different learning components of SCT around healthy eating.

\section{METHOD}

Our study examines how people leverage social platforms throughout their process of food and diet tracking. We interviewed 18 past and present users of food journaling technology to understand how people's use of journaling and social technology intersects and evolves with their eating goals. Prior to the interviews, we examined the features of nine popular Chinese food journaling apps that ranked in top 200 free health \& fitness apps in the Chinese App store with ratings over $1 \mathrm{k}$, including the four that our participants had used (Mint, Food Storehouse, iCalorie, Good Body Knowledge). The apps included similar features to food journaling apps popular in Western society like MyFitnessPal, MyNetDiary, and MyPlate. All four apps that participants used included databases for looking up food information such as calories, the ability to track both calories and nutrients, and suggestions on healthy eating tips. Three included features to set a daily or weekly eating goal, and two included barcode scanning. All four food journaling apps included a dedicated built-in social platform where people could share their food logs. Notably, two apps allowed for other types of sharing in their internal social platforms, where people shared images of how their body had changed, their personal experiences with healthy eating, weight loss progress, or the exercises they were doing.

\subsection{Participants}

As we witnessed an increasing popularity of food journaling apps in China [14], we initially aimed to explore the increasing use of food journaling apps in China to understand how apps' design choices influence people's diet behaviors. In our interviews, participants often mentioned their learning behaviors and the use of various external social platforms during and after food tracking practices when aiming to change their diet behaviors and reach different goals, leading us to focus on this aspect of the interviews. 
We extend findings from our prior work [55], interviewing 18 participants who are currently or have previously used a food journaling app. All participants lived in China during the time of the study. We recruited all participants online, mainly through Chinese social media. We posted our recruitment message on these social media platforms on the first author's WeChat and Weibo's personal timeline, in WeChat and TenCent QQ's public chat groups, and to Weibo's public channels about weight loss or food journaling. We also directly messaged Weibo users who mentioned the name of a popular Chinese food journaling app in a recent post. We additionally invited users who posted frequently on food journaling apps' internal social platforms. Two participants (P16 and P17) were recommended by P13 after the interview. Interviews were about an hour and a half on average. We compensated our participants with $140 ¥$ (about \$20).

Our participants tended to be young (average 25 years, min 20, max 29). Over half of the participants used Mint (10/18), which is the most downloaded and the highest ranked food journaling app in the Chinese App store. Nine participants were students (four undergraduate and five graduate), while the rest ranged in professions from sales, government, art, and telecommunications. Six participants have or had tracked their food for three months or less, five had tracked between three months and a year, and seven had tracked for over a year. All had behavior change or self-improvement goals for food journaling, either to lose weight or gain muscle mass. Seven participants described themselves as actively food journaling, while four indicated they had stopped using food journaling apps. The remaining seven participants described their journaling as intermittent. Table 1 describes our participant demographics.

Table 1. Demographic information about participants in our interview study.

\begin{tabular}{|l|l|l|l|l|l|l|}
\hline ID & Gender & Age & Profession & App used & App use duration & Journaling motivation \\
\hline P1 & Female & 21 & Undergraduate Student & Mint & $1-2$ months & Lose weight \\
\hline P2 & Female & 24 & Content operation & Mint & 6 months-1 year & Lose weight \\
\hline P3 & Female & 24 & Graduate student & Mint & $1-2$ months & Bodybuilding and lose body fat \\
\hline P4 & Female & 25 & Graduate student & Mint & $3-6$ months & Lose weight and healthy eating \\
\hline P5 & Female & 24 & Graduate student & Mint & $3-6$ months & Lose weight \\
\hline P6 & Female & 22 & Graduate Student & Mint & $2-3$ years & Bodybuilding \\
\hline P7 & Male & 23 & Graduate Student & Mint & $2-3$ years & Lose weight and gain muscle \\
\hline P8 & Male & 27 & Research Assistant & Food storehouse & 5 years & Lose weight \\
\hline P9 & Female & 28 & Salesperson & Mint & 4 years & Lose weight \\
\hline P10 & Male & 29 & Scriptwriter & Mint & $3-6$ months & Lose weight \\
\hline P11 & Female & 25 & Product Manager & Mint & $1-2$ year & Lose weight \\
\hline P12 & Male & 31 & Artist & Good body knowledge & $3-6$ months & Lose body fat \\
\hline P13 & Male & 27 & Government Employee & iCalorie & 6 months-1 year & Bodybuilding and lose body fat \\
\hline P14 & Female & 20 & Undergraduate Student & Food storehouse & $3-6$ months & Lose weight \\
\hline P15 & Female & 22 & Undergraduate Student & iCalorie & $1-2$ months & Lose weight \\
\hline P16 & Male & 29 & Insurance Clerk & iCalorie & 6 months-1 year & Gain muscle \\
\hline P17 & Male & 26 & Telecommunications & iCalorie & $3-6$ months & Lose weight \\
\hline P18 & Female & 20 & Undergraduate Student & Food Storehouse & $1-2$ months & Lose weight \\
\hline
\end{tabular}

\subsection{Data Collection}

We conducted interviews synchronously in Mandarin online through WeChat, a popular instant messaging app in China. The researcher sent questions in text, and participants responded with voice memos. The researcher also sent screenshots of app features for participants to react and 
respond to. We chose this mode because WeChat is primarily a text-based platform, we felt participants would prefer to answer a question while looking at it. Having participants respond in voice reduced participant effort and preserved emotions. A few participants occasionally chose to type their responses during the interview. The first author transcribed the voice memos.

Our interview questions fell into two main categories. We first sought to understand people's use of food journaling apps, asking questions like "how has your food journaling app supported your goals?", "what about your goal has your food journaling app not supported as well?", and "How do you perceive a particular feature?'. We then examined people's use of social technologies as social features, such as built-in social platforms, are often present in Chinese food journaling apps , asking questions like "what social technologies have you used?", "why did you use these social technologies for your goal?" and "what did you do on different social platforms for your goal?", and "what did you find useful about the social platform?".

\subsection{Data Analysis}

We analyzed the interview data through a combination of inductive and deductive approaches. Initial open coding was inspired by the grounded theory approach [20] that two authors, both fluent in Mandarin, read the first 10 interview transcripts to get a primary understanding of the data. The two authors open coded these interview transcripts to identify potential themes. After meeting to discuss these themes, we developed nine initial codes around participants' perspectives on app features and use of them. When discussing the data as a research team and comparing with prior literature, we realized that (1) participants frequently mentioned learning behaviors on the social platforms they used during and after food journaling, and (2) participants described use of these social platforms as both intertwined with and evolving from their self-tracking practices. We therefore switched to a more deductive approach by using existing theories and models to help analyze data. Because participants frequently mentioned learning by tracking and on social platforms, we used three relevant components of Social Cognitive Theory [5] (observational learning, enactive learning, self-efficacy) as a deductive codebook to help us make sense of how tracking and social technology support different learning behaviors during behavior change. Our participants' use of food journaling apps resembled the different tracking stages described in the Lived Informatics Model [28] but with strong connections to social technologies. We therefore deductively utilized the Lived Informatics Model [28] to begin to develop our new model, contextualizing our participants' evolving use of social platforms and their learning behaviors on both tracking apps and social platforms while they aimed to change their food behaviors.

With these theoretical lenses in mind, the entire research team met weekly to discuss the findings and further iterate on and refine the codes. Our final list contained 13 parent codes, which we applied to all interviews: Social Cognitive Theory (enactive learning, social learning, and selfefficacy), social media selection, social media influence, social support, social tracking, social sharing, cultural influences on journaling, body image, motivations for journaling, data entry challenges, food tracking practices, perspectives on app's internal social platform, and perspectives on app's built-in eCommerce features. The first author then read all interview transcripts and used the codebook to label quotes related to each parent code. The author grouped quotes with the same parent code into subgroups through affinity diagramming, generating 34 child codes to categorize people's different behaviors or attitudes toward each parent code. For example, two child codes for social tracking contrasted tracking on internal social platforms with tracking on external social platforms, and food tracking practices contained three child codes considering short-term use, 
long-term use, and transitioning from short-term journaling to long-term occasional use. We translated representative quotes for each theme into English after we completed analysis.

\section{MODEL OF SOCIALLY SUSTAINED SELF-TRACKING FOR FOOD AND DIET}

Based on our results, we introduce a Model of Socially Sustained Self-Tracking for food and diet (Figure 1) to describe how people's evolving use of technology between tracking systems and social systems towards different eating goals, expanding on Epstein et al.'s Lived Informatics Model [28]. Our model emphasizes how social platforms inform or intersect with the tracking process to constitute a technology ecosystem for facilitating people's eating and diet goals. Our model also incorporates the enactive learning and observational learning concepts from SCT [5], with tracking and social technology differently supporting people's learning for positive behavior change.

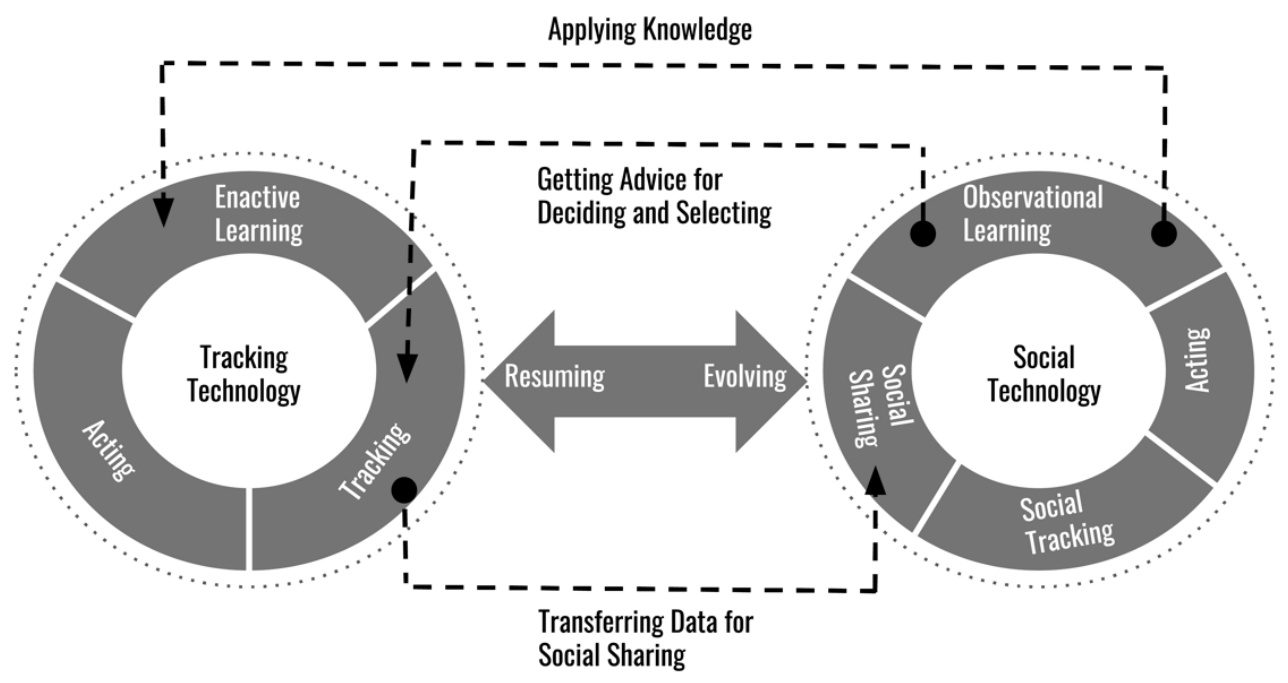

Figure 1. Our Model of Socially Sustained Self-Tracking for food and diet. The left circle shows people's use of tracking technologies, while the right circle shows people's use of social technology like social networking sites, messaging apps, and Q\&A sites. The evolving and resuming arrows illustrate how people's use of technology transitions between tracking technology and social platforms. The dashed line arrows (transferring data for social sharing, applying knowledge from social platforms, and getting advice from social platforms) show how people's practices and experiences tracking and using social platforms influence one another. As in the Lived Informatics Model [28], all the activities in the circles occur simultaneously in practice, including one circle's influence on the other. For example, people often act, learn, and track (including transferring data to social platforms) simultaneously when using tracking technology.

The left circle of our model represents the cycle of tracking illustrated in the Lived Informatics Model: deciding \& selecting; tracking \& acting; lapsing; resuming [28]. Our findings extend this process to suggest that people aim to learn about the tracking domain alongside tracking and acting. We therefore visually simplify the life cycle of tracking in the Lived Informatics Model, showing and extending its "Tracking \& Acting" stage. The right circle represents people's use of external social platforms for behavior change and self-improvement, showing that they use those technologies to learn by observation, socially track, socially share, and act based on these 
interactions simultaneously. People evolve and migrate between circles, such as evolving to external social platforms after stopping to track to achieve deeper social or learning goals and resuming tracking when necessary to answer specific domain or self-knowledge questions. Our model also highlights three ways people use social technology while self-tracking: (1) As described in the Lived Informatics Model, people sometimes get advice from social platforms which influences their choice of tracking app or tool [28]. (2) Aligned with previous studies on social sharing [16,27], our model reflects that people transfer their tracked data or progress their data represents to external social platforms for goals such as instrumental tracking, being held accountable, and to offer or receive emotional support. (3) People also apply knowledge gained from social platforms about their habits or ways to improve their habits to their tracking practices.

Overall, our model highlights that the use of tracking apps is part of an ecosystem where use of social apps informs, evolves from, and coexists with tracking. We next illustrate how the left circle (the use of tracking apps) and the right circle (the use of external platforms) mutually influence each other in people's broader use of technology.

\section{RESULTS}

To explain our Model of Socially Sustained Self-Tracking, we elaborate on how participants' use of technology for tracking, sharing, and learning evolve as their eating goals progress. Participants' lived experience of tracking was similar to processes described by the Lived Informatics Model [28]. They decided to track and selected tools to support eating goals, tracked and acted by collecting and reflecting on their data, and lapsed in tracking while occasionally resuming. We discovered social sharing behaviors similar to prior studies, such as providing and receiving social support, instrumental tracking, and facilitating communication [2,16,27,65]. We therefore do not describe our participants' practices which align closely with the Lived Informatics model, and only briefly highlight sharing practices which align with prior personal informatics systems. Participants' reflective practices around their data aligned with practices described in past models $[9,28]$. We focus on describing people's learning via observation and action rather than reflection that has been less surfaced in the literature. Further work unpacking how reflection does or does not relate to different learning behaviors, building on recent work on data-mediated reflection $[8,9,30]$ can further extend our understanding of the role of self-tracking towards learning. In presenting our model, we instead focus on describing how people's learning behaviors shape their use of both tracking technologies and social platforms, highlighting how people leverage internal and external social platforms, such as using social platforms while tracking to learn domain knowledge and practices for reaching eating goals, and the evolving from tracking to using dedicated social platforms to sustain behavior change and to obtain more specific domain knowledge. We also introduce how people's self-efficacy is enhanced or decreased by different social technology.

We highlight four kinds of social technologies (Table 2) which intersected with participants' use of tracking technology that participants mentioned during the interview, and describe popular analogs in Western social media: 
Table 2. Description of the Chinese social technologies mentioned in interviews.

\begin{tabular}{|l|l|}
\hline Types of Social Technology & Specific Social Platforms \\
\hline Social networking platforms & $\begin{array}{l}\text { Weibo (similar to Twitter) } \\
\text { Xiaohongshu (similar to Instagram, used primarily by female participants) } \\
\text { Tieba (similar to Reddit) }\end{array}$ \\
\hline Social messaging apps & $\begin{array}{l}\text { WeChat and Tencent QQ (similar to WhatsApp or any messaging platform). } \\
\text { Besides direct messaging, these two apps allow people to create group chats and submit posts on their } \\
\text { news feed. }\end{array}$ \\
\hline Video sites & $\begin{array}{l}\text { Douyin (similar to Tiktok) and Bilibili (similar to YouTube). } \\
\text { Douyin is used for creating and sharing short videos for social interaction, while longer videos are shared } \\
\text { on Bilibili. }\end{array}$ \\
\hline Question and answer (Q\&A) sites & Zhihu (similar to Quora) \\
\hline
\end{tabular}

\subsection{Getting Advice While Deciding \& Selecting}

Participants' decision to track and process of selecting tools were influenced by information they acquired from friends or online strangers on social platforms. Participants expressed two major motivations to track their daily diet: to have a more satisfying body figure or to become healthier by improving their diet. Participants' goals would sometimes shift from achieving a particular body image (usually becoming slimmer) to overall health as they journaled their food. For example, P5 said, "the biggest motivation is to lose weight. Then it actually turned into maintaining a healthy body". Notably, some participants' goals changed during the tracking process: "at first I used the app to lose weight since I wanted to control calories, but later I found that the effect was not so obvious. However, now my main purpose is to be healthy. There are things that you really can't consume too much, which are bad for the body and your body can't digest them well' (P4).

Similar to in the Lived Informatics Model [28], some participants got help selecting a particular food journaling app from their friends via social messaging apps or influencers they followed on social networking platforms. P7 said, "I once weighed about $320 \mathrm{lbs}$ and all my friends said I should lose weight. Then they recommended me to use Mint". Information on various social platforms, mostly social networking platforms, video sites, and Q\&A sites, portrayed an ideal body image that led some participants to become dissatisfied with their own body figure, aligning with prior work that social media significantly influences young women's concerns about body image [68]. This led some participants to seek out food journaling apps to reach their ideal body weight. P3 complained that "sometimes I feel that words on the internet make people feel it is an original sin to be fat". P2 called herself an "aesthetic deformity" when she saw Kpop idols' pictures on the internet, making her believe that "the slimmer the better". People's practices aligned with the motivation component of observational learning, by which people's intention or motivation to change is influenced by what they see in others [5]. This socially acquired information, whether positive recommendations of apps or negative perceptions of body image caused by social platforms, all contributed to participants' motivation of using food journaling apps and defining their goals.

\subsection{Transferring Tracking Data to Social Platforms for Social Support}

Participants often leveraged external social platforms to support different sharing behaviors while they tracked. Specifically, participants described using their tracked data to facilitate similar social interactions to what previous work has described, such as receiving and providing support [16,25], showing accountability [16], instrumental sharing [2,65], and facilitating communication [36]. For example, P10 shared screenshots of his food logs on WeChat's news feed or directly messaged his friends. Though his initial intention was to "show off" his weight loss progress, he realized sharing 
food data helped promote accountability and help him receive support, making him become more persistent in keeping a healthy diet and losing weight: "When you say something publicly, you then must be persistent in it to not be discreditable. So (Sharing) works as a monitoring and encouragement'. The sharing behavior also triggered topics around body training between P10 and his friends: "I also find that when you have some improvements (on eating and losing weight), people are more willing to discuss these things with you. You'll suddenly notice that some of your friends are doing the same thing". Participants also sometimes shared their food data or progress to motivate and educate their friends, similar to findings in past work [7,51]. For example, P6 constantly shared pictures of healthy foods she made and found delicious to Tencent or WeChat to teach her friends about dieting: "My perception about diet is to make it both delicious and healthy. There's not much difference between normal people and people with training goal. The latter one just eats a bit healthier than normal people. You don't need to only eat chicken breast for training. Healthy diet needs to be close to normal food, in this way you can be persistent in it longer".

Participants also described using food logs to facilitate similar accountability and instrumental practices to past work [12,59,78], such as collectively monitoring weight loss and exchanging diet practices. Many people in China use the term “check-in (打卡)" to describe the way in which they report their daily activities on social media. P9 shared her daily food log from Mint to a weight loss WeChat group, to be held accountable, but also to share what she's eating: "the check-in is quite useful. Because on WeChat, we communicate more. We will communicate what ingredients we need to eat as well as our weight loss experience. The app Mint is used as a tool. That is, we record what we eat today by using Mint, and then share these records to our WeChat group for check-in".

\subsection{Learning while Tracking}

Through our interviews, participants repeatedly mentioned how their tracking practices are entangled with efforts to learn, suggesting that learning is a fundamental goal and outcome of people's food tracking process. Furthermore, tracking tools, their internal social platforms, and external social platforms all contributed to people's learning but in different ways. Aligned with learning concepts of SCT [5], food logging features in tracking tools provided an enactive learning experience while social platforms offered observational learning.

\subsubsection{Enactive Learning from Tracking Tools}

Common food journaling features such as feedback on what they entered, information provided by food databases, and tutorials for healthy eating practices allowed participants to gain selfknowledge and some basic domain knowledge through enactive learning. Though information provided by these features were primarily used to regulate and maintain people's newly-built diet behaviors, participants were able to learn the potential course of action which was crucial for achieving certain healthy eating or weight managing goals when using these features. When participants first started tracking, they were particularly interested in understanding their own habits and adjusting their behaviors based on the goal set by the food journaling app, such as learning the suggested daily consumed calories based on their demographics and goal. For example, P15 said, "once I exceed the intake, I will exercise to burn the extra calories, so that calories journaled by the app won't exceed". Goal-setting features also enabled participants to learn general knowledge about the human body, such as understanding terms like Body Mass Index (BMI) and body fat rate. P1 claimed that "before using the app, I never noticed the glycemic index of the food. I only knew BMI but I didn't know the body fat rate. I have learned a lot of new knowledge and have a 
more scientific perception of body image". Feedback from food journaling apps, such as analysis of intake, allowed participants to learn about the calories and macronutrients (e.g., protein, fats, carbohydrates) of the foods they ate to help them plan and select what to eat. For example, P4 said " $m y$ favorite feature is to search and check calories in the database. Whenever I do groceries, if I really want to eat something and even a lot of food, I would check all these food's calories to see which one has the lowest calories. Then I would choose that one to eat". Some apps also contained instructions, such as workout tutorials or articles, to raise users' awareness of doing exercises and teach them how to exercise properly. P14 claimed, "this app will tell you what kind of exercise you can do to burn calories and how long it will take to burn them. I think this is very helpful to me".

Our data showed that enactive learning contributed to participants' observational learning by raising their health awareness, which related to the attention component of observational learning. Before tracking their food, most participants described having limited knowledge of their habits, of what constituted healthy eating, and of best practices in other related health domains such as exercise. For example, P17 said, "I used to have no clear perceptions about eating and I didn't even know what calories mean. After using the food journaling app and when I started to lose weight, then I changed my perceptions toward diet". P10 even claimed the food journaling app "inverted" his knowledge about eating: "it was my first time knowing how much calories that a bowl of rice contains and how much I need to exercise to burn these calories". Participants also described previously struggling to achieve their goals because they lacked necessary domain knowledge, such as P1: "I usually paid close attention to my diet but I still didn't have much progress in losing weight. So I want to see if my daily diet is not reasonable enough (by using the food journaling app)". Participants felt basic self and domain knowledge acquired via journaling food had changed their prior perceptions around diet, making them pay more attention to the importance of healthy diet and other related practices. As P17 said, "The app has indeed changed my perceptions about diet a lot. I used to think that if I eat meat, I would gain weight. Then, I discovered that carbon will affect your weight loss effect. If you simply eat some protein like meat, you won't gain fat. The app indeed explains some things about diets". P1 similarly expressed how acquiring necessary knowledge help attain her goal: "The app made me pay attention to daily diet by knowing calories of common food and their nutrition information. I also would do some exercises. Therefore, the effect is pretty good". Consequently, participants began proactively seeking more domain knowledge on external social platforms. As their health awareness had been raised, both P17 and P1 leveraged different external social platforms to acquire more knowledge through observation, such as "following people who do great in body training and people who provide guidance on diet on Douyin [a short-video platform]" (p17) or “following professionals' WeChat [a social messaging app] public account" (p1).

\subsubsection{Observational Learning from Internal and External Social Platforms While Tracking}

While tracking, the built-in social platforms of Chinese food journaling apps enabled participants to learn socially from both reading what others would post and interacting with others to get their specific diet and eating habit questions answered. Through observing others' posts, participants mainly "get inspiration about how to eat" (P2) and learn others' weight-loss methods. P15 said, "if I see the body shape shared by other users is the one I want, I would click her account and read the sports she shares as well as her own experience". The built-in social platforms allow people to reflect on their own methods by seeing other user's food diaries and experiences. For example, P2 said, "I could know whether my way of losing weight is scientific or not by seeing the food and experiences shared by other people". Participants also used the social feeds to ask people they follow for advice 
or clarification. P1 said, "I would follow them when I see someone who provides a good experience. Then if I have any questions about the post, I would directly comment under the post. Generally, people would answer my questions except some who have a lot of fans".

Participants also learned from social networking platforms while they tracked, particularly picking up healthy recipes from video sites, and Q\&A sites. For example, P9 used Weibo to search for low-fat but tasty recipes when using the food journaling app to lose weight because she wished to know "how to make fat-reducing meals more delicious". Participants felt these learning needs cannot be satisfied by food journaling apps and their built-in social platform, typically because built-in platforms did not have significant enough activity or information. P2 said, "take Xiaohongshu [similar to Instagram] as an example, it has a lot of users on it and then a lot of things are shared while less things on the food journaling app's built-in social platform. If I'm looking for a recipe or something, I'll definitely want to find it on Xiaohongshu instead of the food journaling app".

Participants' learning from social platforms involved three observational learning components described in SCT: attention, production, and motivation [5]. Pictures posted by other users on social platforms could easily attract participants' attention: "People with good body shapes are quite attractive. I would follow people on Weibo who have good body shapes” (p13). In addition, social platforms supported participants' selective attention by allowing them to freely choose what knowledge or practices they were interested in and what form of knowledge they would like to learn from. This support was enabled by social platform's convenient search features and rich media options (e.g., short posts with pictures, articles, and videos) as well as the variety of existing social technology. For example, P9 leveraged Weibo to "search for a new weight loss method", Tieba to "find people with similar interests and backgrounds in losing weight" for social support, and Bilibili to "watch videos" about weight-loss practices. Towards production, knowledge shared on social platforms made participants understand the potential course of action for achieving particular goals. For example, social platforms provided participants with specific and actionable plans towards their goals by obtaining healthy eating concepts, diet recipes, and workout tutorials. P15 described that she "obtained the nutrition knowledge from Weibo by knowing what food in daily life has low calories. I would then choose to eat these food". Social platforms also offered a space for participants to perform what they have learned: "I write posts on my WeChat public account to summarize what knowledge I have learned about health as well as my own experiences long since [I started my goal]" (P11). Finally, information observed on social platforms motivated some participants to realize the importance of healthy eating. For example, P13 said, "When I first started losing weight [by the food journaling app], I valued my appearance a lot. I felt it was important to lose weight to have a good body shape. Then, nutrition knowledge [learned on external social platforms] makes me realize that eating is as important as appearance. I more and more realize the importance of healthy eating and being healthy". P13 then started to pay more attention to healthy eating, saying that he would "buy books", "watch videos on Bilibili", "talk with a coach" to systematically learn nutrition and body training.

\subsection{Evolving to Social Platforms}

Participants typically evolved to external social platforms to continue or sustain their behavior change process when tracking no longer served their needs. When participants first started tracking, most were passionate about logging food and learning about the calories they consumed daily. P8 was so enthusiastic when first using the app to journal food that "I checked whatever I ate, even at night. I also checked the calories whenever I thought of snacks". However, most participants 
only persistently journaled for a short amount of time. For example, both P17 and P5 expressed that they only diligently tracked food for a month or two. Then participants began to lapse and even abandon tracking due to similar reasons uncovered by previous studies [24,28], such as no need to track or the fading of tracking motivation. Most of our participants gained sufficient self and domain knowledge through journaling. For example, P8 felt no need to continue logging food daily because "you won't need to continuously check the same food". Some participants, such as P11, even said "[I] have built a fairly strong knowledge of nutrition in this process of journaling" that they can "have a very good control of my diet". Previous studies mainly attribute people's abandonment of tracking to either successfully reaching goals (e.g., "happy abandonment") or frustrated tracking experiences, such as tracking burden, losing curiosity to track, concerns about sharing data [28]. A few of our participants did abandon the app as they approached their ideal weight. P13 stopped journaling after he successfully lost weight: "the reason for me to stop using the app is not because I got used to it. It is my current goal is not to reduce fat, so I don't need this app".

\subsubsection{Reasons for Evolving}

Participants had five main reasons for evolving to use external social platforms: satisfying role models, deeper domain knowledge, more reliable domain knowledge, richer social support, and a like-minded audience. For most participants, the motivations for weight loss or increased muscle mass that led them to track their food persisted even after they lapsed or abandoned tracking. With food journaling apps no longer supporting their evolving needs well, many participants moved on to external platforms to continue their behavior change process.

Participants' desires for further learning, sharing, and tracking informed their choices about which kind of social platform(s) to evolve to. Participants mainly used social messaging apps and social networking platforms to acquire richer support and find like-minded audiences. Participants looking to gain deeper and more reliable domain knowledge used all four types of systems.

Finding Satisfying Role Models. Having a role model demonstrate good behaviors is useful for observational learning because people learn knowledge and perform corresponding behaviors by observing them [5,60]. The design features of external social platforms such as recommendation, and search features, and a larger user base helped participants find knowledgeable or inspirational role models. For example, participants appreciated how external social platforms recommended similar people to those that they followed: "I followed some professional coaches or other people who are good at fitness training. When you follow them, Weibo would recommend similar people to you and I would immediately follow them" (P16). Additionally, participants chose people with different backgrounds and skills as their models from the large audience on external social platforms. Participants followed professionals or experts to gain foundational scientific knowledge, normal people who could provide actionable instructions on healthy eating and weight loss, and people who had body shapes or other healthy habits they wished to emulate on different social platforms.

Role models on external social platforms supported participants by increasing their self-efficacy and facilitating the observational learning processes of attention, production, and motivation. Seeing others' success in reaching the same goals they had helped participants enhance their own beliefs: "Social media have changed my beliefs when I saw people on Bilibili could reduce fat efficiently by good fitness training and healthy eating"(P13). Similarly, as discussed in the previous section, P10 realized more friends were "willing to discuss these things [about healthy eating and weight loss] with you" when he "had some improvements [on eating and losing weight]". 
Richer Social Support. Our participants expressed similar social needs as described in prior studies, such as seeking out or receiving support [16]. They typically chose to seek out external social platforms over internal social platforms, though previous studies show that tracking tools often leverage social features to keep users engaged [19,31,50]. Current Chinese food journaling apps typically include social feeds, inviting others to also register the app, and features for sharing food logs on external social platforms, to satisfy people's social needs. Our participants felt that the social support that the food journaling apps offered was insufficient and thus preferred external social platforms.

Participants seemed to primarily leverage social networking platforms and social messaging apps to gain support with pre-existing social contacts or like-minded online strangers. Notably, Weibo and WeChat are two major platforms when participants mentioned interacting with others and getting support. First, these two apps are the most commonly-used social apps in China, compared with other social technologies that were mentioned in the interview, that participants feel it's convenient to reach out to others and get feedback since "people are now using WeChat all the time that you can get timely communication on WeChat (P9)". Second, though some did receive support from like-minded online strangers, most participants tend to gain social support from their pre-existing social networks. P4 compared WeChat with Xiaohongshu, a social networking platform they used to connect with people they do not know: "one (WeChat) is to socialize with acquaintances while another (Xiaohongshu) is to socialize with strangers. I prefer to interact with acquaintances because it's more real and I would like to let these people know my privacy". Interestingly, some participants did not follow their acquaintances on Weibo, and they would explicitly share their data on WeChat: "both Xiaohongshu and Weibo are to socialize with strangers while WeChat is to socialize with acquaintances. I only want to share what exercises I did and what food I ate with my acquaintances (P8)".

Besides receiving social support, some participants also aimed to provide social support and motivate others by sharing their experience or progress on WeChat or Weibo, similar to previous work [16]. P7 said, "I posted my weight loss experience and body pictures on Weibo with my friends to tell them 'I am thinner'. I also want to use this to motivate some of my friends, who are also overweight, to lose weight together'. Participants' offering support to others reflected the modeling and production in observational learning. Participants became models when sharing the knowledge they had obtained and useful personal experiences to "wish my friends to pay attention to health" (P18). Similarly, P11 wrote posts about healthy eating on her public account to "help others and influence people around me". P11 would "tell some daily healthy eating knowledge from the aspect of nutriology. Take my parents as an example, I would tell them how to pay attention to eating at their age". For people with weight loss goals, she would "provide some professional guidance by making simple diet recipes and teaching simple knowledge". Participants also demonstrated what they had learned by sharing the experiences and knowledge they gained during the behavior change process. Through educating others, participants demonstrated that they had internalized what course of action they should take to achieve their goals.

However, compared with external social platforms, food journaling apps and their built-in social platform were unable to satisfy participants' desire for social support. Most participants felt their food journaling app was predominantly a tracking tool to reach their weight loss or healthy eating goals, even though it has a built-in social platform. P9 said, "I deem the food journaling app as a tool that allows me to search for food and journal it. The external social platform is what I use to communicate every day". Although journaling app's social features provide opportunities for users 
to interact with others, participants still preferred to interact with people on external social platforms. This was true for both interacting with pre-existing social relationships or online strangers with similar interests. For example, P4 had privacy concerns around the built-in social platform "[it] doesn't have much privacy to chat when there are a lot of strangers and it doesn't give me a strong sense of security". In addition, participants felt the small user base of internal social platforms made it difficult to get responses compared to external social media. P12 compared the internal social platform with Weibo: "in terms of promoting knowledge, I think the influence of Weibo is much greater than these food journaling apps because more people are using Weibo now in China. As for Good Body Knowledge [one food journaling app], its users are very limited".

Participants also felt posting pictures on built-in social platforms was more burdensome since they believed that these pictures needed to highlight healthier practices than ones posted to external platforms. For example, P17 said, "for Wechat's Moments and Weibo, you usually would follow some bloggers you like, and your friends will also follow your account as well as seeing your Wechat's Moments. In this case, you usually would communicate about your daily life on these social platforms. In the case of the food journaling app's social platform, you rarely meet people you know and most users are people you want to learn from or think they are good. The internal platform is more learning-oriented, and observation-oriented, which is not a platform for communication. There will be less communication on it, mostly are your learning process".

Although social comparison is often regarded as beneficial for sustaining engagement with tracking tools and facilitating self-improvement [48,50], our participants tended to feel less positive about social comparison features of built-in social platforms as their self-efficacy could be decreased. For example, P9 found the built-in social platform of her app frustrating because it often showed posts from other users whose body figures felt unattainable: "Well, as for some of the people the app recommended to me, they are fitness pictures of beautiful women. Then I didn't think there was a need for me to lose weight. Uh, I feel that we don't have the same topics. These people are so thin. I would also feel upset since they already have very good body shapes but still are doing exercises. There are no people with the same body figure as me. I feel I am the fattest person in the world and unhappy".

A Like-minded Audience. Our participants desired getting social support from people with similar body types and weight loss goals, feeling that interacting with them could build a sense of community [16,57]. Aligning with previous studies [16], participants felt external social platforms were better able to help them interact with whoever they want to, such as offline acquaintances or online strangers with the same goal or interests, than internal social platforms. Participants tended to leverage social networking platforms, especially Weibo, rather than social messaging apps to seek like-minded audiences. For example, when P9 was searching for weight loss knowledge on Weibo, she first followed people who organized the weight-loss "check-in" WeChat group. Furthermore, participants who were interested in specific fitness goals such as bodybuilding found it difficult for them to get support from their pre-existing social networks, and instead used external social media to find a community with a similar identity. For example, P6 was upset that her friends did not understand her interest, "I basically posted pictures about my body figure on my Weibo instead of WeChat, because few acquaintances on Weibo. Very few girls that I know are also doing fitness training and they don't understand your aesthetic. They would feel that you look good when wearing clothes. But when seeing your muscles, they will think "Ah, you are so strong"'?. Hence, she would share pictures of food she enjoyed, but were low-calorie with her offline acquaintances on WeChat while using Weibo to share body training pictures with her 
workout friends. Interacting with like-minded audiences helped participants increase their selfefficacy, aligning with the vicarious experience in self-efficacy that people's belief in their ability to successfully perform a behavior can be enhanced by seeing the success of others, especially people who are like themselves [5,60]. For example, P9 believed she could lose more weight when observing that "other people [like-minded audiences] could lose weight" successfully.

Participants' use of social networking platforms or social messaging apps for communicating with like-minded audiences would typically evolve as they interacted. They would often start with basic interactions such as commenting on others' posts and/or following others' accounts. Then, some moved to WeChat for further interaction and more in-depth communication, such as direct conversations or forming a WeChat group. For example, P12 would "ask other questions about long-distance running on Weibo or get to know someone who also runs very well. Then I would add their WeChat to talk more".

Deeper domain knowledge. Participants felt tracking was primarily useful for obtaining basic dietary knowledge, and stopped once they are familiar with these basic knowledge. For example, P2 believed that "I now have a relatively good understanding of what to eat and what not to for weight loss" through the process of tracking her diet. However, other participants wished to learn more detailed or professional knowledge about food to support their goals. For example, P13 says that "people, like me and my friends, would learn the knowledge of nutrition, fitness on our own initiative. What we need more is to learn this knowledge systematically and apply this". P7 even "learned and passed the test of the Registered Dietitian when I was losing weight". For these participants, their desire to learn more made them unsatisfied by the limited knowledge provided by their food journaling app. P11 felt that the "knowledge provided by the diet app is too shallow". Therefore, these hungry-for-knowledge users evolve to external platforms to continue reaching their goals.

Participants felt that external platforms provided multiple ways to acquire domain knowledge beyond reading posts which introduce domain knowledge. P11 learned in-depth knowledge on Weibo by “seeing followers' questions and professor Fan's answers because Professor Fan will interact with her followers. You can even ask questions yourself. But on Mint, there is no one that you can ask questions to". P3 acquired domain knowledge on Zhihu, a Q\&A site, by "checking answers about people's own process or experience about fitness and weight-loss". Some participants watched video tutorials or other people's experiences on Douyin and Bilibili. P8 prefers Bilibili to learn fitness knowledge because "many fitness tutorials there and it's quite convenient to find". Video sites and Q\&A sites primarily supported participants in learning about fitness and weight-loss techniques. Participants learned knowledge in other areas from Weibo by following experienced users or experts. For example, P6 mainly learned bodybuilding knowledge by "follow fitness experts who participated in bodybuilding competitions". P11 learned professional dietary and exercise knowledge by "follow [ing] professionals such as fitness coaches and yoga coaches. I will also follow dietitians like Professor Fan". P2 mainly used Weibo to "search for what I should eat when I want to lose weight".

External platforms typically present information in richer forms than data shared in food journaling apps, leveraging words and images alongside GIFs, short and long videos. P12 chooses Weibo to gain knowledge because "many things are shared on Weibo, including videos, words, GIFs, or articles". Prior work shows that videos' visual and auditory stimuli can contribute to people's better understanding of concepts when learning [76]. Therefore, P9 felt videos were effective for educating weight-loss methods and workout tutorials because "people will summarize their 
experiences in just one video and I can directly learn some important knowledge completely in just one video". P10 summarized how and what external social media supported their learning: "Some KOLs [Key Opinion Leaders on social media] on social media will teach some scientific and rational knowledge. Also, there are some materials like videos and images. All these things could slowly change my perceptions about healthy lifestyle, body shape, and fitness".

More Reliable Domain Knowledge. In addition to people's feeling that the domain knowledge provided by the app can be shallow, some participants also felt that the app, particularly its internal social platform, was sometimes a source of incorrect or misleading information from unprofessional sources. P7 said, "people who are in charge of sharing knowledge on the food journaling app are definitely not someone who are professional. Or maybe the app has professional coaches, but the person who directly spreads the information must not be a professional person". Some participants pointed out that the app may even educate users with unhealthy or even harmful weight-loss methods. P7 complained that "The food journaling app overemphasizes the perception that it is good to have abs and a strong body figure through training. This will cause a one-sided effect, making people feel overwhelmed. Sometimes the app provides a kind of guidance that is not healthy". P6 has a similar opinion: "Mint had been scolded before because it once advocated people to do fasting”. P2 had a negative experience when interacting with a user who was primarily interested in selling weight-loss products: "I usually just observe others' interactions. When I first lost weight, I once added a person's WeChat account, but then I found out that she was selling diet pills. Since then, I don't believe these things. I think I'll just follow my own way". P15 even felt that "I always feel users on Weibo are more real. Users on the iCalorie [a food journaling app] are not real".

Participants typically achieved trustworthy knowledge by either actively following reliable role models or searching platforms for desired knowledge. P7 explained how nutritionists on the external social platform Weibo, instead of users on the food journaling app's built-in social platform, helped him build healthy eating habits: "it is Professor Fan [a famous Chinese professor in Nutrition Science that the participant follows on Weibo] who tells me that if you want to reduce calories, but you must supplement the reduced calories with healthy food, and your body figure will be gradually improved to a very good one instead of a fat and weak state". Similar to Twitter, Weibo enables people to verify their expertise and professional training by submitting related materials such as diplomas, professional certificates, portfolios, and publications. Once verified, the verification will then appear on the verified user's profile with a yellow "V" icon. The verification gave participants a sense of whether someone is a trustworthy expert or not. As a result, P11 believed that "I know she [Professor Fan] has professional certificates. She has all kinds of things that I don't doubt" since Professor Fan is verified as "Director of China Nutrition Society, director of China Association for Health Promotion and Education, Doctor of Food Science" on her profile. P11 further compared Professor Fan with people who provided knowledge on food journaling apps: "as for the app Mint, I can't see who is writing the knowledge shared on it and I don't know who the app's nutritionist is. He/She doesn't seem like a living person like Professor Fan, who gives me an authoritative feeling. Hence, Weibo must be more reliable than 'I-CANNOT-SEE-PEOPLE' Mint'.

Additionally, external platforms provide easier mechanisms for searching and finding reliable information sources than internal ones. P7 tends to "follow some public accounts of reliable fitness bloggers on Zhihu". P18 follows bloggers interested in different aspects relating to wellbeing on different sites: "I follow people who share cooking on Xiaohongshu and fitness on Weibo". P2 would use both Weibo and Xiaohongshu to "search what should be eaten when losing weight". P12 prefers 
Weibo to learn because "there are actually many things that are shared on Weibo. More extensive information on Weibo and more professional coaches (compared with the food journaling app)".

Overall, the diversity of platforms and their affordances make external platforms more valuable for learning than social platforms in food journaling apps. Participants' preferences of how and what they wanted to learn influenced their platform choice.

As a summary, these five reasons for evolving to external social platforms indicated that these platforms could better satisfy people's deeper and diverse social and learning needs than tracking tools and internal social platforms. The design features of external platforms (e.g., search mechanism, recommendation, and verification) enabled individuals to more easily find role models, like-minded audiences, and reliable domain knowledge aligned with their goals. In addition, the multitude of external social platforms and their broader audiences provided in-depth domain knowledge in different forms and catered to people's evolving needs for social support. As a comparison, internal social platforms tended to decrease people's self-efficacy in reaching their goals through social comparison, lowering their willingness to share and seek social support.

\subsection{Resuming the Act of Food Journaling}

Participants occasionally returned to their food journaling app briefly to acquire more information the journal was well-suited to provide. For example, participants sometimes revisited the app when encountering unknown food. P11 said, "actually, I no longer record my diet and I use it for searching. Because in the process of using the app, I have built a fairly strong knowledge of nutrition, and then I have a very good control of my diet that I don't need to use the app for journaling. In fact, I probably know how many calories I consume in a day and whether the calories are reasonable. I will only check the app when I encounter something that I don't know its calories". P5 also transitioned from short-term persistent use of food journaling to the long-term occasional resumption: "at the beginning, I journaled the food diligently for a month or two. Now I will only use the app to search the calories of some foods which I am not sure".

People would also resume whenever their circumstances or goals change such that food journaling would help them again. For example, P9 said food journaling was most useful when they were pursuing a weight loss goal: "the reason why I don't use Mint recently is because I am not determined to lose weight. I usually use the app when I want to lose weight'. P13 expressed a similar opinion, saying, "now my current plan is that I will use this app when there is a need for reducing fat or gaining muscles. I will not use the app if there is no need. Because I will follow a strict diet that if I use the food journaling app, I will weigh the food. It is indeed very complicated and brings some troubles to my life. Well, I can't say troubles, just there is not so much time often".

Notably, even when resuming food tracking apps for either acquiring knowledge or pursuing goals, participants still leveraged external platforms for learning, tracking, sharing, and acting. Resumption of tracking technology was a "quick check", where people only returned to the tracking app to know food that they were not familiar with.

\section{DISCUSSION}

Our Model of Socially Sustained Self-Tracking expands on prior personal informatics models [28,47] by highlighting where tracking intersects with social technologies to develop an ecosystem in support of the long-term and complex process of behavior change. Built on empirical findings in the food journaling domain, our model depicts people's lapsing and abandonment of tracking tools as an 
evolving process, showing how they appropriate other platforms to take further action or maintain positive behavior change. Our result further presents how different kinds of social platforms were preferred by people to satisfy different goals, such as providing and receiving support from acquaintances or strangers with similar goals on social messaging apps, identifying like-minded audiences on social networking platforms, following satisfying role models for observational learning, and finding reliable domain knowledge on social networking platforms, Q\&A and video sites. Our model also describes how people flow between using tracking and social technology to achieve their social interaction and self-knowledge goals.

Our model also highlights the significance of enactive learning in the tracking practice, suggesting that "Tracking \& Acting" in the Lived Informatics Model [28] can be thought of as "Tracking, Acting, \& Learning" in the eating domain. Figure 2 describes how the enactive and observational learning components of SCT relate to the use of tracking and social technology described by our model. People's learning needs often motivate their adoption of tracking technology and drive them to engage with other platforms to receive deeper or more reliable knowledge. When tracking, food journaling apps enable people to enactively learn via the process of journaling food, while social platforms let people to learn by observing others. When self-tracking technology no longer support people's learning, people evolve to social technology to satisfy deeper learning needs. After evolving to social technologies, some people may occasionally resume tracking as a "quick check" to acquire some knowledge which is easier to get from the tracking technology itself. While previous literature $[28,47]$ has mostly discussed how tracking tools can help people gain self-knowledge, we also find that tracking tools can provide some basic domain knowledge through features such as databases with information about foods or activities, feedback that apps provide on eating reports, and included how-to guides or articles. People often then turn to social platforms to gain more domain knowledge, but can struggle to find trustworthy knowledge sources.

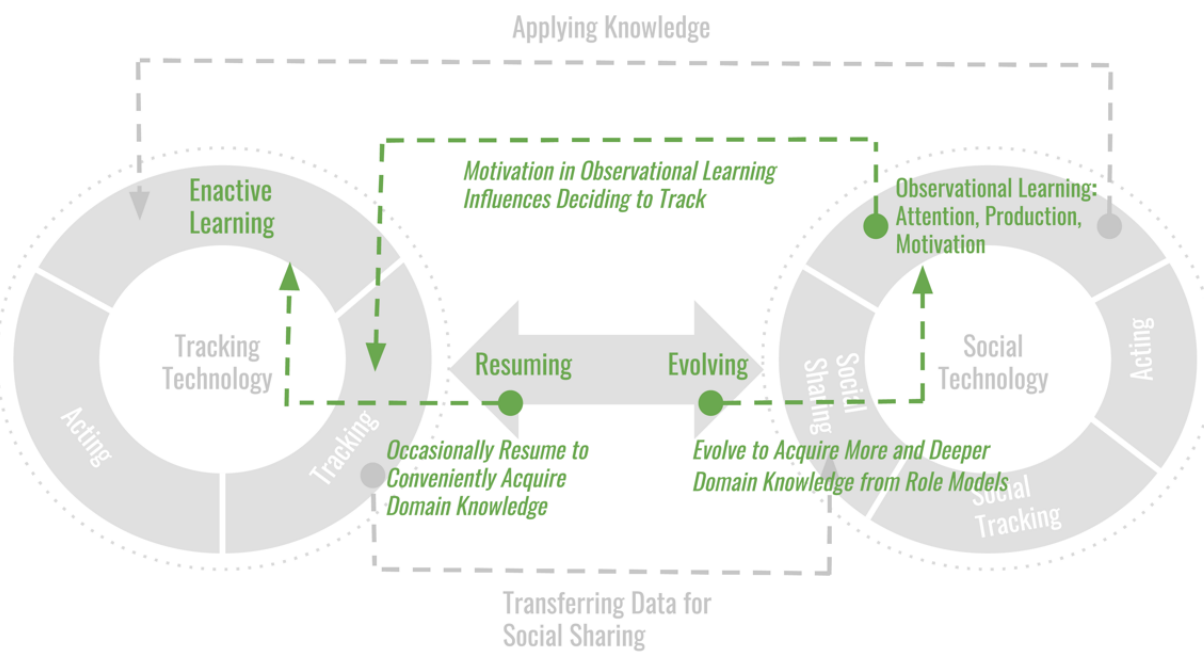

Figure 2. Our model surfaces how enactive learning and observational learning in SCT relate to people's use of technology to build healthy eating habits. We find that tracking apps enable people to conveniently gain basic domain knowledge when searching and journaling food, while external social platforms allow them to gain more and deeper domain knowledge through observation. People resume tracking or evolve to use social technology based on their knowledge needs, and are occasionally motivated to decide to track from observing others. 
We now discuss the implications of our model on our understanding of the relationship between tracking technology and social technology, supporting sustainable behavior change, considering the role of social technology in other tracking domains, and improving food technologies.

\subsection{Rethinking Lapsing and Abandonment of Tracking as a Gateway to Social Technology}

Similar to the Lived Informatics Model [28], our findings suggest people lapse in food journaling and stop tracking once they have achieved or abandoned their goals for tracking. This stopping is sometimes considered perceived as negative, with some people stopping tracking because the data collected was not useful for self-improvement, maintaining the habit was too difficult, and managing data was complex [45,73]. Our study surfaces that people who are particularly passionate about acquiring domain knowledge typically stop tracking when their learning needs can no longer be satisfied by the tracking app. They then evolve to use external social platforms to continue learning new knowledge and sustaining their process of behavior change via observation. Our data suggests that external social platforms are more helpful for strengthening self-efficacy than social features within tracking technology. These findings all demonstrate the role of tracking technologies as "gateway" tools [72] to social technologies to facilitate people's evolving needs when trying to change behavior. Considering the typical short-term engagement with tracking technology $[38,58]$ and people's inevitable evolving needs when behavior change progresses, we suggest that tracking should not be simply regarded as a self-contained activity that aims to solely support initiating goals, reaching them, and maintaining progress. Viewing tracking technology as a gateway could additionally lead to less frustration or guilt when stopping tracking [24].

Our study also shows that people occasionally return to food tracking when they desire new food related knowledge. Instead of considering lapsing as an abandonment of the technologies, burdened by tracking or achieving tracking goals, what we found in the study identify a new, positive form of lapsing. This suggests people's tracking goals are not static, but rather evolving as their knowledge about the tracking domain as changed. This positive connotation towards lapsing and abandonment shows how behavior change should be thought of as embedded in the larger ecosystem of technology use, and efforts to sustain long-term tracking should be considered in light of people's evolving use to social technologies. Although research has commonly discussed approaches to designing for sustained engagement with personal informatics tools $[33,37,45]$ our work surfaces a need to consider how to design tracking tools as an effective gateway to other technologies, in particular social technologies.

\subsection{Selecting and Leveraging Social Platforms for Sustainable Behavioral Change}

Our model also articulates how people's use of tracking tools and social platforms for eating intersect and mutually influence one another. Much like our finding that people leverage social platforms to gain necessary information to help take action during tracking, Thackeray et al. [79] found that people appropriated social media to seek additional information, such as consulting online reviews about drugs or treatments. When seeking recommendations on social platforms, Teodoro \& Naaman [78] suggested that people search for others with similar interests, lurking and gaining inspiration from what others are doing. However, our study found that participants questioned the reliability of the recommendations they saw from built-in social platforms in food journaling apps, seeking to learn deeper and more reliable domain knowledge from experts on external social media. Since participants had prior self- and domain-knowledge from having 
tracked their food, they typically evolved to external social platforms with a clearer sense about what knowledge or recommendations they desired and from whom they could get it from on social platforms. Having a clearer learning goal, participants selected different social platforms to acquire the knowledge they need. Some chose a particular social platform because of its reliable experts and experienced users, such as P11 seeking out and following the famous dietitian Professor Fan on Weibo. Some participants chose a particular social platform because knowledge on it is presented in a form that is efficient and convenient to learn, such as workout tutorials and videos of people's weight-loss experiences on video sites.

Our participants sought out audiences with similar interests when sharing data on social platforms, echoing prior work [16,61]. Previous studies suggested that people proactively build and shape their audience by inviting like-minded people from online communities to their Facebook [61] or selectively share their data with a subgroup on Facebook (e.g., by building their own Facebook groups) [27]. However, rather than insisting on one social platform, our participants additionally considered which of the social platforms they were familiar with would best facilitate the kind of social support they desired. This finding aligns with previous work suggesting that people consider both content and audience appropriate when choosing how to manage and share content on multiple social platforms $[74,85]$. People intentionally choose social platforms to control the audience being reached. In our study, participants had different concerns or aims when sharing socially such as trusting their audience, being held socially accountable, seeking encouragement from acquaintances, and finding like-minded audiences to gain a sense of identity. As a result, participants preferred to share their diet and weight-loss progress with their pre-existing social contacts on social messaging apps, while sharing fitness data with like-minded audiences on social networking platforms. Furthermore, since participants leveraged social platforms not only for sharing but also for observational learning, we discovered that different social channels were selected based on their affordances. Direct messaging channels, such as social messaging apps, are better at initiating social support and enhancing relationships as they enable people to have indepth two-way communication. Broader channels, such as social networking platforms, video sites, and Q\&A sites, are better for facilitating observational learning as they allow people to find and follow experts, professionals, and like-minded audiences.

Participants' preference towards external social platforms for observational learning and social needs also reflects how social technology influences people's self-efficacy, a key attitudinal factor that forecasts the success or failure of behavior change [71]. Though prior studies often incorporate and find value in social features into tracking systems [7,50,75], our findings suggest that external social platforms do better in enhancing self-efficacy. External social platforms allowed participants to choose the right people to engage with, either observing the practices of role models for learning or interacting with like-minded audiences for social support. By contrast, social comparisons in internal social platforms, such as sharing successes or experiences which appear unattainable, occasionally decreased participants' self-efficacy. Participants deliberately selected certain social platforms to resonate with the audiences they wished to engage with and be motivated by.

\subsection{Generalizing the Model of Socially Sustained Self-Tracking to Other Domains}

Although we describe our model in the specific case of food and diet tracking, we believe many of the relationships between tracking tools and social platforms exist in other domains where people frequently track. The Lived Informatics Model illustrates that people often turn to friends and family members for advice on what tools to use to track physical activity, location, and finances 
[28]. Studies of online health communities such as around fertility care [22], menopause [46], weight loss [49], and chronic disease [42] surface that people often reach out to like-minded groups to self-track and share, aiming for emotional support and recommendations for behavior change and health management. Kaye et. al [40] further report on users' desire for more domain knowledge to help them better manage their finances in the future, similar to our participants' learning needs in the food domain. Social platforms around finance have similarly supported observational learning, with newcomers learning about social and cultural norms around sending and receiving money by observing others' activities shared online [12]. Studies of tracking physical activity and finances have surfaced that people often stop tracking when they learn enough about what they need to act, obtain the necessary skills to do so, or develop plans for doing so [24]. Though similar social and/or learning needs are found in other tracking domains, to generalize the model, future work is still needed to test and validate this model in these domains.

However, we expect that differences between tracking domains likely limit how well our model describes how social platforms intersect with tracking technology in other domains. The Lived Informatics Model suggests that people often receive tracking tools as gifts, which decides tool selection [28]. Social media may provide less advice on tool selection in domains where tracking is done with a physical device, such as physical activity as people often receive physical trackers as gifts $[28,70]$. All of our participants sought to improve their eating practices, and the desire to acquire self- and domain-knowledge is likely more prevalent among people tracking with behavior change goals. However, for people who are motivated to track out of a desire to have a record or for curiosity, this knowledge may be less essential. Informing and interacting with others is a typical motivator for people to seek out location tracking technology [28]. People often seek out location tracking technology with robust internal social platforms, rather than evolving their practices to social-first platforms. In addition, some people and domains may not have as deep of domain learning needs. For example, location tracking technology and social interaction tracking technology are primarily driven by the instrumental goal to record a particular behavior and share data to initiate social interaction, rather than learning domain knowledge [28,52].

Cultural context may also influence the model's application. Evolving to social technology was particularly emphasized by our participants. Their desire to use social media in their behavior change process might have been influenced by collectivist culture, which emphasizes strong social ties [39]. However, we suspect that evolving to use social media is likely to extend beyond the Chinese context. Prior studies in Western contexts have shown people's strong social needs in tracking tools and the use of external social platforms [26,27,78]. The pervasive use of social technologies in Western culture may exert a similar influence on people's tracking behaviors.

\subsection{Design Opportunities}

Our findings suggest opportunities for tracking technology and social platforms to better serve people's healthy eating behavior change process. We acknowledge that these suggestions cannot address all challenges surfaced in our work, particularly in circumstances where food journaling apps are helping to promote negative or unhealthy eating habits [21,23]. These design opportunities offer potential suggestions to help make the complex technology ecosystem easier to navigate and more comprehensively support people's goals. Our design opportunities focus on two aspects: (1) leveraging tracking tools as a gateway to better support people's observational learning behaviors on external social platforms, and (2) facilitating people's evolving from tracking technologies to social technologies. 


\subsubsection{Leveraging Food Journaling Apps as a Gateway for Observational Learning}

Participants were strongly interested in learning domain knowledge from external social platforms. However, as open platforms that almost everyone can share their opinions, social media can offer conflicting or harmful information alongside reliable knowledge. Some participants found it difficult to interpret whether the practices for healthy eating that they read on social media were trustworthy. Some also struggled to manage the extreme perceptions of ideal body image disseminated on social platforms, aligning with past work showing social media's role in facilitating community and practices around eating disorders [13,23,66]. Substantial research has explored challenges around regulating misinformation spread on social media (e.g., [81]). Though our findings suggest that food journaling apps cannot satisfy people's observational learning needs for acquiring in-depth and specialized knowledge, they could serve as a gateway, arming people with accurate perceptions toward healthy eating and body image. As our findings suggest that basic knowledge gained through journaling food could contribute to the attention component of observational learning, the more explicitly food journaling apps present necessary domain knowledge, the more that people using food journals would have greater awareness of their health and the more their goals might evolve. For example, food journals could use the initial setup, goalsetting and feedback features in tracking to discuss healthy eating concepts and positive perceptions about body image, incorporating references to scientific studies or knowledge. Current apps mostly focus on goal setting and tracking features around calories, while they seldom contain features that explicitly guide people on how to eat healthily. In terms of the production component of observational learning, the databases in food journaling apps could incorporate meal planning features to provide a specific course of action for healthy eating. Many users, such as P4, would search for food's calories in the databases to decide whether to eat or purchase it, rather than only logging the food after consumption. Apps could therefore recommend healthy recipes or other people's related posts when searching one food or ingredient in the database. The enactive learning experience of food journaling apps could be also combined with the reflection stage of self-tracking [9,28,30]. For example, in addition to providing knowledge explicitly, apps could also prompt people to reflect on their current eating behaviors, which may invite changes in eating behaviors.

Food journaling apps could also identify and critique unrealistic or dangerous ideas about eating, health, and body image on social media. Weibo once promoted a challenge where young females held an A4 sheet paper in front of their waists to show how slim they were [54]. Similarly, challenges around body image often go viral on TikTok in Western society. Although some challenges message body positivity, people often still comment on the appearance of challenge participants [41]. Our work suggests that trends like these could drive people to adopt food journaling apps. Whenever a challenge or trend goes viral on social media, experts could be invited to the food journaling platform to discuss potential risks surrounding the trend within the journal's internal social platforms.

Although participants typically viewed food journaling apps as trustworthy sources of information, some participants questioned the reliability of the information presented. For example, P11's app included articles on best dieting practices and scientific understanding of food, but users doubted these articles because "I can't see who is writing the knowledge shared on it and I don't know who the app's nutritionist is". Therefore, food journaling apps could increase its transparency and reliability by providing background on the writer's name, occupation, and contact information. Other tracking apps such as Clue incorporate references to scientific literature in their articles [80]. 


\subsubsection{Supporting Evolving from Food Journaling Apps to Social Platforms}

Aligning with prior work [16], many participants shared their food journals socially prior to evolving to use social platforms more exclusively. Exporting tracked data from food journaling apps to external social platforms could be improved by customizing the content of shared data to the different types of social platforms people are interested in sharing with. Our participants tended to seek for like-minded audiences on social networking platforms such as Weibo. When exporting data on Weibo, the food journaling app could recommend hashtags used by people with varied diet and eating goals, enabling their posts to be seen by like-minded audiences. However, when sharing data on WeChat's Moments (a user's feed), the app could instead help surface to preexisting social contacts what kinds of responses are desired, perhaps incorporating WeChat stickers such as "asking for praise". However, sharing these data sometimes can be regarded as trivial and be ignored by selected audiences [27]. People's passion for sharing food journaling data socially could be decreased if they don't receive a desirable level of social feedback. Tensions around who is receiving tracked data and how likely they are to offer support or encouragement should all be considered when incorporating such a feature.

Tracking apps could also better help people evolve to social platforms by identifying channels or communities where experts or like-minded people are discussing specific goals. For example, our participants preferred to learn fitness tutorials on video sites such as Douyin and Bilibili. Food journaling apps could incorporate articles or other features which introduce professional coaches which provide reliable fitness tutorials on these platforms, with links to their accounts and videos. Similarly, for people who have a particular interest in accumulating more professional and scientific dietary knowledge, the app could suggest trustworthy dietitians on Weibo or Zhihu. For people who wish to identify recipes which are healthy while tasty, the app could suggest corresponding food influencers on Weibo and Xiaohongshu.

These design suggestions are unlikely to make either food journaling apps or social apps allencompassing to provide one-stop experiences that satisfy people's overall learning, tracking, and acting needs during the entire behavior change process. Designing with the expectation that people will stick to a single app or platform can undermine people's flexibility to choose platforms which have the most convenient or reliable access to the audiences they want to share with or learn from. Social platforms internal to tracking apps can be useful for helping people who are collecting similar data learn from one another's experiences, but they may struggle to support the range of goals or depth of social support of the ecosystem of social-only platforms. Internal social platforms inherently will not enable people to reach the critical mass of people that dedicated social platforms allow, and are often unable to access the close ties who people often want to share with. We therefore suggest that apps promote exporting of data and facilitate identifying appropriate social platforms for more in-depth knowledge or like-minded audiences, rather than presuming that an improved internal social platform will be able to facilitate all of people's social needs.

\subsection{Limitations}

By recruiting participants via social media, our study demographics skewed young (between 20 and 30 years old). Our participants were likely more interested in social platforms as an opportunity for support and knowledge. Older participants or those less engaged with social media may not evolve their tracking technology use to social platforms as readily. Over half of our participants used Mint (10/18). Although this aligns with the relative popularity of the app compared to other food 
journaling apps in China, we acknowledge that the features present in or absent from other apps may lead to different tracking motivations or evolutions to other tools.

By recruiting participants who were still somewhat engaged in food journaling, our participants primarily "happily abandoned" food journaling technology [24], learning enough about their eating habits or about best practices for healthy eating prior to using social platforms. However, prior work suggests that people also lapse or abandon tracking technology because they find it too burdensome or because their goals change [24,28]. Our findings therefore do not capture how people's technology use evolves if they abandoned journaling because they found it too burdensome or if they abandoned their self-improvement goal altogether. These burdens and goal abandonment may be more common in other tracking domains, such as ones which require maintenance of tracking devices (e.g., wearable devices for tracking physical activity).

Video conferencing software which allowed attendees to share screens, such as Zoom or Skype, were not particularly popular in China at the time of the study. Because we asked participants to view app screenshots during our interview, we conducted interviews with WeChat's voice memo feature since WeChat's video call feature cannot share screens. We acknowledge that interviews were less efficient since the researcher had to first play the participant's voice memo, then to ask follow-up questions based on the participant's response. We took care to ask probing questions in spite of this limitation, but the long interviews may have been more fatiguing for participants and reduced willingness to share their experiences.

Though our study examines the benefits that food journaling apps and social platforms brought during people's behavior change process in our interviews, we want to acknowledge that prior work has surfaced potential risks and harms of food journaling and social media on people's eating habits [23,81]. Some of our participants described similar experiences where social media influenced them to negatively judge their own physical health.

\section{CONCLUSION}

We present a new personal informatics model in the food and diet tracking domain that illustrates how tracking technologies and social technologies are integrated as an ecosystem to facilitate people's long-term behavior change process. Our model surfaces that observational learning, and a desire for deeper and more reliable domain knowledge, often motivates people to evolve from tracking technologies to social platforms. We suggest that tracking technology can be looked at as a "gateway" to other technology for behavior change, and tracking technology can further support people's natural evolution by recommending social groups or platforms which might match their changing needs. We caution against trying to fully integrate social platforms into tracking tools to support people's evolution, as convenience and access to the right audience for the moment make maintaining such an audience challenging.

\section{ACKNOWLEDGMENTS}

We thank Elena Agapie and our anonymous reviewers for their feedback. This work was funded in part by the National Science Foundation under award IIS-1850389.

\section{REFERENCES}

[1] Tawfiq Ammari and Sarita Schoenebeck. (2015). Understanding and Supporting Fathers and Fatherhood on Social Media Sites. Proceedings of the SIGCHI Conference on Human Factors in Computing Systems (CHI 2015), 1905-1914. http://doi.org/10.1145/2702123.2702205 
[2] Amid Ayobi, Tobias Sonne, Paul Marshall, and Anna L. Cox. (2018). Flexible and Mindful Self-Tracking: Design Implications from Paper Bullet Journals. Proceedings of the SIGCHI Conference on Human Factors in Computing Systems (CHI 2018), 28-41. http://doi.org/10.1145/3173574.3173602

[3] Albert Bandura. (2004). Health Promotion by Social Cognitive Means. Health Education and Behavior 31, $143-164$. http://doi.org/10.1177/1090198104263660

[4] Albert Bandura. (2001). Social Cognitive Theory of Mass Communication. Media Psychology 3, $265-299$. http://doi.org/10.1207/S1532785XMEP0303_03

[5] Albert Bandura. (1986). Social Foundations of Thought and Action: A Social Cognitive Theory. Prentice-Hall, Inc. https://psycnet.apa.org/record/1985-98423-000

[6] Albert Bandura and Richard H. Walters. (1977). Social Learning Theory. Prentice-hall Englewood Cliffs, NJ.

[7] Eric P.S. Baumer, Sherri Jean Katz, Jill E. Freeman, Phil Adams, Amy L. Gonzales, John Pollak, Daniela Retelny, Jeff Niederdeppe, Christine M. Olson, and Geri K. Gay. (2012). Prescriptive Persuasion and Open-ended Social Awareness: Expanding the Design Space of Mobile Health. Proceedings of the ACM Conference on Computer Supported Cooperative Work (CSCW 2012), 475-484. http://doi.org/10.1145/2145204.2145279

[8] Marit Bentvelzen, Jasmin Niess, Mikołaj P. Woźniak, and Paweł W. Woźniak. (2021). The Development and Validation of the Technology Supported Reflection Inventory. Proceedings of the SIGCHI Conference on Human Factors in Computing Systems (CHI 2021), 366-373. http://doi.org/10.1145/3411764.3445673

[9] Marit Bentvelzen, Jasmin Niess, and Paweł W. Woźniak. (2021). The Technology-Mediated Reflection Model: Barriers and Assistance in Data-Driven Reflection. Proceedings of the SIGCHI Conference on Human Factors in Computing Systems (CHI 2021), 1-12. http://doi.org/10.1145/3411764.3445505

[10] Brandon Brown, Marshini Chetty, Andrea Grimes, and Ellie Harmon. (2006). Reflecting on Health: A System for Students to Monitor Diet and Exercise. Proceedings of the SIGCHI Conference on Human Factors in Computing Systems (CHI 2006), 1807-1812. http://doi.org/10.1145/1125451.1125794

[11] Eleanor R. Burgess, Renwen Zhang, Sindhu Kiranmai Ernala, Jessica L. Feuston, Munmun De Choudhury, Mary Czerwinski, Adrian Aguilera, Stephen M. Schueller, and Madhu C. Reddy. (2020). Technology Ecosystems: Rethinking Resources for Mental Health. Interactions, 28(1), 66-71. http://doi.org/10.1145/3434564

[12] Monica Caraway, Daniel A. Epstein, and Sean A. Munson. (2017). Friends Don't Need Receipts: The Curious Case of Social Awareness Streams in the Mobile Payment App Venmo. Proceedings of the ACM on Human-Computer Interaction, 1(CSCW), 17. http://doi.org/10.1145/3134663

[13] Stevie Chancellor, Jessica Annette Pater, Trustin Clear, Eric Gilbert, and Munmun De Choudhury. (2016). \#Thyghgapp: Instagram Content Moderation and Lexical Variation in Pro-Eating Disorder Communities. Proceedings of the ACM Conference on Computer Supported Cooperative Work (CSCW 2016), 1201-1213. http://doi.org/10.1145/2818048.2819963

[14] Chanpinyibai. User Experience Report of the App Mint. http://www.kejilie.com/chanpin100/article/3UZF3i.html

[15] Eun Kyoung Choe, Nicole B. Lee, Bongshin Lee, Wanda Pratt, and Julie A. Kientz. (2014). Understanding Quantified-Selfers' Practices in Collecting and Exploring Personal Data. Proceedings of the SIGCHI Conference on Human Factors in Computing Systems (CHI 2014), 1143-1152. http://doi.org/10.1145/2556288.2557372

[16] Chia-Fang Chung, Elena Agapie, Jessica Schroeder, Sonali Mishra, James Fogarty, and Sean A. Munson. (2017). When Personal Tacking Becomes Social: Examining the Use of Instagram for Healthy Eating. Proceedings of the SIGCHI Conference on Human Factors in Computing Systems (CHI 2017), 1674-1687. https://doi.org/ccq4

[17] James Clawson, Jessica A. Pater, Andrew D. Miller, Elizabeth D. Mynatt, and Lena Mamykina. (2015). No Longer Wearing: Investigating the Abandonment of Personal Health-Tracking Technologies on Craigslist. Proceedings of the ACM International Joint Conference on Pervasive and Ubiquitous Computing (UbiComp 2015), 647-658. http://doi.org/10.1145/2750858.2807554

[18] Mark Conner and Paul Norman. (2005). Predicting Health Behaviour: Research And Practice With Social Cognition Models. Open University Press.

[19] Sunny Consolvo, Katherine Everitt, Ian Smith, and James A. Landay. (2006). Design Requirements for Technologies that Encourage Physical Activity. Proceedings of the SIGCHI Conference on Human Factors in Computing Systems (CHI 2006), 457-466. http://doi.org/10.1145/1124772.1124840

[20] Juliet Corbin and Anselm Strauss. (2014). Basics of Qualitative Research: Techniques and Procedures for Developing Grounded Theory. SAGE Publications, Inc.

[21] Felicia Cordeiro, Daniel A. Epstein, Edison Thomaz, Elizabeth Bales, Arvind K. Jagannathan, Gregory D. Abowd, and James Fogarty. (2015). Barriers and Negative Nudges: Exploring Challenges in Food Journaling. Proceedings of the SIGCHI Conference on Human Factors in Computing Systems (CHI 2015), 1159-1162. https://doi.org/bbdt

[22] Mayara Costa Figueiredo, Clara Caldeira, Tera L. Reynolds, Sean Victory, Kai Zheng, and Yunan Chen. (2017). Self-Tracking for Fertility Care: Collaborative Support for a Highly-Personalized Problem. Proceedings of the ACM on Human-Computer Interaction, 1(CSCW). http://doi.org/10.1145/3134671 
[23] Elizabeth V. Eikey and Madhu C. Reddy. (2017). "It's Definitely Been a Journey": A Qualitative Study on How Women with Eating Disorders Use Weight Loss Apps. Proceedings of the SIGCHI Conference on Human Factors in Computing Systems (CHI 2017), 642-654. http://doi.org/10.1145/3025453.3025591

[24] Daniel A. Epstein, Monica Caraway, Chuck Johnston, An Ping, James Fogarty, and Sean A. Munson. (2016). Beyond Abandonment to Next Steps: Understanding and Designing for Life After Personal Informatics Tool Use. Proceedings of the SIGCHI Conference on Human Factors in Computing Systems (CHI 2016), 1109-1113. http://doi.org/10.1145/2858036.2858045

[25] Daniel A. Epstein, Felicia Cordeiro, James Fogarty, Gary Hsieh, and Sean A. Munson. (2016). Crumbs: Lightweight Daily Food Challenges to Promote Engagement and Mindfulness. Proceedings of the SIGCHI Conference on Human Factors in Computing Systems (CHI 2016), 5632-5644. https://doi.org/bjh8

[26] Daniel A. Epstein, Mira Dontcheva, James Fogarty, and Sean A. Munson. (2020). Yarn: Adding Meaning to Shared Personal Data through Structured Storytelling. Proceedings of Graphics Interface (GI 2020), $168-182$. http://doi.org/10.20380/GI2020.18

[27] Daniel A. Epstein, Bradley H. Jacobson, Elizabeth Bales, David W. McDonald, and Sean A. Munson. (2015). From "Nobody Cares" to "Way to Go!": A Design Framework for Social Sharing in Personal Informatics. Proceedings of the ACM Conference on Computer Supported Cooperative Work (CSCW 2015), 1622-1636. https://doi.org/bbks

[28] Daniel A. Epstein, An Ping, James Fogarty, and Sean A. Munson. (2015). A Lived Informatics Model of Personal Informatics. Proceedings of the ACM International foint Conference on Pervasive and Ubiquitous Computing (UbiComp 2015), 731-742. http://doi.org/10.1145/2750858.2804250

[29] Giannina Ferrara, Jenna Kim, Shuhao Lin, Jenna Hua, and Edmund Seto. (2019). A Focused Review of Smartphone Diet-Tracking Apps: Usability, Functionality, Coherence with Behavior Change Theory, and Comparative Validity of Nutrient Intake and Energy Estimates. JMIR mHealth and uHealth, 7(5). https://doi.org/gg82dw

[30] Rowanne Fleck and Geraldine Fitzpatrick. (2010). Reflecting on Reflection: Framing a Design Landscape. Proceedings of the Conference of the Computer-Human Interaction Special Interest Group of Australia on ComputerHuman Interaction (OZCHI 2010), 216-223. http://doi.org/10.1145/1952222.1952269

[31] Thomas Fritz, Elaine M. Huang, Gail C. Murphy, and Thomas Zimmermann. (2014). Persuasive Technology in the Real World: A Study of Long-term Use of Activity Sensing Devices for Fitness. Proceedings of the SIGCHI Conference on Human Factors in Computing Systems (CHI 2014), 487-496. http://doi.org/10.1145/2556288.2557383

[32] Jeana Frost and Brian K. Smith. (2003). Visualizing Health: Imagery in Diabetes Education. Proceedings of the ACM Conference on Designing for User Experiences (DUX 2003), 1-14. http://doi.org/10.1145/997078.997094

[33] Rúben Gouveia, Evangelos Karapanos, and Marc Hassenzahl. (2015). How Do We Engage with Activity Trackers? A Longitudinal Study of Habito. Proceedings of the ACM International foint Conference on Pervasive and Ubiquitous Computing (UbiComp 2015), 1305-1316. http://doi.org/10.1145/2750858.2804290

[34] Andrea Grimes and Richard Harper. (2008). Celebratory Technology: New Directions for Food Research in HCI. Proceedings of the SIGCHI Conference on Human Factors in Computing Systems (CHI 2008), 467-476. http://doi.org/10.1145/1357054.1357130

[35] Joan E. Grusec. (1992). Social Learning Theory and Developmental Psychology: The Legacies of Robert Sears and Albert Bandura. Developmental Psychology. http://doi.org/10.1037/10155-016

[36] Xinning Gui, Yu Chen, Clara Caldeira, Dan Xiao, and Yunan Chen. (2017). When Fitness Meets Social Networks: Investigating Fitness Tracking and Social Practices on WeRun. Proceedings of the SIGCHI Conference on Human Factors in Computing Systems (CHI 2017), 1647-1659. http://doi.org/10.1145/3025453.3025654

[37] Rebecca Gulotta, Jodi Forlizzi, Rayoung Yang, and Mark Wah Newman. (2016). Fostering Engagement with Personal Informatics Systems. Proceedings of the ACM Conference on Designing Interactive Systems (DIS 2016), 286-300. http://doi.org/10.1145/2901790.2901803

[38] Elina Helander, Kirsikka Kaipainen, Ilkka Korhonen, and Brian Wansink. (2014). Factors Related to Sustained Use of a Free Mobile App for Dietary Self-Monitoring with Photography and Peer Feedback: Retrospective Cohort Study. Fournal of Medical Internet Research, 16(4). http://doi.org/10.2196/jmir.3084

[39] Geert Hofstede. (2011). Dimensionalizing Cultures: The Hofstede Model in Context. Online Readings in Psychology and Culture, 2(1), 8. http://doi.org/10.9707/2307-0919.1014

[40] Joseph 'Jofish' Kaye, Mary McCuistion, Rebecca Gulotta, and David A. Shamma. (2014). Money Talks: Tracking Personal Finances. Proceedings of the SIGCHI Conference on Human Factors in Computing Systems (CHI 2014), 521530. http://doi.org/10.1145/2556288.2556975

[41] Mona Khattab. (2019). Synching and Performing: Body (Re)-Presentation in the Short Video App TikTok. WiderScreen, 21(1-2), 1-15. http://widerscreen.fi/numerot/2019-1-2/synching-and-performing-bodyrepresentation in the short video -app-tiktok/

[42] Michael Von Korff, Jessie Gruman, Judith Schaefer, Susan J. Curry, and Edward H. Wagner. (1997). Collaborative 
Management of Chronic Illness. Annals of Internal Medicine. https://doi.org/ggchnx

[43] Robert Kraut, Michael Patterson, Vicki Lundmark, Sara Kiesler, Tridas Mukopadhyay, and William Scherlis. (1998). Internet Paradox: A Social Technology that Reduces Social Involvement and Psychological Well-Being? The American psychologist, 53(9), 1017-1031. http://doi.org/10.1037//0003-066X.53.9.1017

[44] Liliana Laranjo. (2016). Social Media and Health Behavior Change. In Participatory Health through Social Media. Elsevier Inc., 83-111. http://doi.org/10.1016/B978-0-12-809269-9.00006-2

[45] Amanda Lazar, Christian Koehler, Theresa Jean Tanenbaum, and David H. Nguyen. (2015). Why We Use and Abandon Smart Devices. Proceedings of the ACM International foint Conference on Pervasive and Ubiquitous Computing (UbiComp 2015), 635-646. http://doi.org/10.1145/2750858.2804288

[46] Amanda Lazar, Norman Makoto Su, Jeffrey Bardzell, and Shaowen Bardzell. (2019). Parting the Red Sea: Sociotechnical Systems and Lived Experiences of Menopause. Proceedings of the SIGCHI Conference on Human Factors in Computing Systems (CHI 2019). http://doi.org/10.1145/3290605.3300710

[47] Ian Li, Anind Dey, and Jodi Forlizzi. (2010). A Stage-Based Model of Personal Informatics Systems. Proceedings of the SIGCHI Conference on Human Factors in Computing Systems (CHI 2010), 557-566. https://doi.org/bh8zsb

[48] Jia Li, Xuan Liu, Ling Ma, and Weiqiang Zhang. (2019). Users' Intention to Continue Using Social FitnessTracking Apps: Expectation Confirmation Theory and Social Comparison Theory Perspective. Informatics for Health and Social Care, 44(3), 298-312. http://doi.org/10.1080/17538157.2018.1434179

[49] Victor Li, David W. McDonald, Elizabeth V. Eikey, Jessica Sweeney, Janessa Escajeda, Guarav Dubey, Kaitlin Riley, Erika S. Poole, and Eric B. Hekler. (2014). Losing It Online: Characterizing Participation in an Online Weight Loss Community. Proceedings of the International ACM Conference on Supporting Group Work (GROUP 2014), 35-45. http://doi.org/10.1145/2660398.2660416

[50] James J. Lin, Lena Mamykina, Silvia Lindtner, Gregory Delajoux, and Henry B. Strub. (2006). Fish'n'Steps: Encouraging Physical Activity with an Interactive Computer Game. Proceedings of the International Conference on Ubiquitous Computing (UbiComp 2006), Springer Verlag, 261-278. http://doi.org/10.1007/11853565_16

[51] Janne Lindqvist, Justin Cranshaw, Jason Wiese, Jason Hong, and John Zimmerman. (2011). I'm the Mayor of My House: Examining Why People Use Foursquare - A Social-Driven Location Sharing Application. Proceedings of the SIGCHI Conference on Human Factors in Computing Systems (CHI 2011), 2409-2418. https://doi.org/c956b2

[52] Fannie Liu, Laura Dabbish, and Geoff Kaufman. (2017). Supporting Social Interactions with an Expressive Heart Rate Sharing Application. Proceedings of the ACM on Interactive, Mobile, Wearable and Ubiquitous Technologies (IMWUT 2017). http://doi.org/10.1145/3130943

[53] Fannie Liu, Mario Esparza, Maria Pavlovskaia, Geoff Kaufman, Laura Dabbish, and Andrés Monroy-Hernández. (2019). Animo: Sharing Biosignals on a Smartwatch for Lightweight Social Connection. Proceedings of the ACM on Interactive, Mobile, Wearable and Ubiquitous Technologies (IMWUT 2019), 1-19. http://doi.org/10.1145/3314405

[54] Valeria Lotti. (2018). The Image of the Beautiful Woman: Beauty Ideals in Modern Urban China. ASIEN - The German fournal on Contemporary Asia, 147(April), 92-104. http://doi.org/10.11588/asien.2018.147.14492

[55] Xi Lu, Yunan Chen, and Daniel A Epstein. (2021). How Cultural Norms Influence Persuasive Design: A Study on Chinese Food Journaling Apps. Proceedings of the ACM Conference on Designing Interactive Systems (DIS 2021), 619-637. http://doi.org/10.1145/3461778.3462142

[56] Kai Lukoff, Taoxi Li, Yuan Zhuang, and Brian Y. Lim. (2018). TableChat: Mobile Food Journaling to Facilitate Family Support for Healthy Eating. Proceedings of the ACM on Human-Computer Interaction, 2(CSCW), 114. http://doi.org/10.1145/3274383

[57] Diane Maloney-Krichmar and Jenny Preece. (2005). A Multilevel Analysis of Sociability, Usability, and Community Dynamics in an Online Health Community. ACM Transactions on Computer-Human Interaction, 12(2), 201-232. http://doi.org/10.1145/1067860.1067864

[58] Jochen Meyer, Judy Kay, Daniel A. Epstein, Parisa Eslambolchilar, and Lie Ming Tang. (2020). A Life of Data: Characteristics and Challenges of Very Long Term Self-Tracking for Health and Wellness. ACM Transactions on Computing for Healthcare, 1(11), 1-4. http://doi.org/10.1145/3373719

[59] Sean A. Munson and Sunny Consolvo. (2012). Exploring Goal-Setting, Rewards, Self-Monitoring, and Sharing to Motivate Physical Activity. Proceedings of the International Conference on Pervasive Computing Technologies for Healthcare (PervasiveHealth 2012), 25-32. http://doi.org/10.4108/icst.pervasivehealth.2012.248691

[60] Razieh Tadayon Nabavi. (2012). Bandura's Social Learning Theory \& Social Cognitive Learning Theory. Theory of Developmental Psychology, 1-14.

[61] Mark W. Newman, Debra Lauterbach, Sean A. Munson, Paul Resnick, and Margaret E. Morris. (2011). It's Not that I Don't Have Problems, I'm Just Not Putting Them on Facebook: Challenges and Opportunities in Using Online Social Networks for Health. Proceedings of the ACM Conference on Computer Supported Cooperative Work (CSCW 2011), 341-350. http://doi.org/10.1145/1958824.1958876

[62] Jasmin Niess and Paweł W. Woźniak. (2018). Supporting Meaningful Personal Fitness: The Tracker Goal

PACM on Human-Computer Interaction, Vol. 5, No. CSCW2, Article 451, Publication date: October 2021. 
Evolution Model. Proceedings of the SIGCHI Conference on Human Factors in Computing Systems (CHI 2018). http://doi.org/10.1145/3173574.3173745

[63] Charoula K. Nikolaou and Michael E.J. Lean. (2017). Mobile Applications for Obesity and Weight Management: Current Market Characteristics. International fournal of Obesity, 41(1), 200-202. https://doi.org/f9kf7z

[64] Kiemute Oyibo, Ifeoma Adaji, Rita Orji, Babatunde Olabenjo, Mahsa Azizi, and Julita Vassileva. (2018). Perceived Persuasive Effect of Behavior Model Design in Fitness Apps. Proceedings of the ACM Conference on User Modeling, Adaptation and Personalization (UMAP 2018), 219-228. http://doi.org/10.1145/3209219.3209240

[65] Kunwoo Park, Ingmar Weber, Meeyoung Cha, and Chul Lee. (2016). Persistent Sharing of Fitness App Status on Twitter. Proceedings of the ACM Conference on Computer Supported Cooperative Work (CSCW 2016), 184-194. http://doi.org/10.1145/2818048.2819921

[66] Jessica A. Pater, Oliver L. Haimson, Nazanin Andalibi, and Elizabeth D. Mynatt. (2016). "Hunger Hurts but Starving Works:" Characterizing the Presentation of Eating Disorders Online. Proceedings of the ACM Conference on Computer Supported Cooperative Work (CSCW 2016), 1185-1200. http://doi.org/10.1145/2818048.2820030

[67] Wei Peng. (2009). Design and Evaluation of a Computer Game to Promote a Healthy Diet for Young Adults. Health Communication, 24(2), 115-127. http://doi.org/10.1080/10410230802676490

[68] Richard M. Perloff. (2014). Social Media Effects on Young Women's Body Image Concerns: Theoretical Perspectives and an Agenda for Research. Sex Roles, 71(11-12), 363-377. http://doi.org/10.1007/s11199-014-0384-6

[69] Robert Rockmann and Heiko Gewald. (2018). Activity Tracking Affordances: Identification and Instrument Development. Proceedings of Pacific Asia Conference on Information Systems (PACIS 2018), 232. https://aisel.aisnet.org/pacis2018/232

[70] John Rooksby, Mattias Rost, Alistair Morrison, and Matthew Chalmers. (2014). Personal Tracking as Lived Informatics. Proceedings of the SIGCHI Conference on Human Factors in Computing Systems (CHI 2014), 1163-1172. http://doi.org/10.1145/2556288.2557039

[71] Herman Saksono, Carmen Castaneda-Sceppa, Jessica A. Hofman, Magy Seif El-Nasr, and Andrea G. Parker. (2021). StoryMap: Using Social Modeling and Self-Modeling to Support Physical Activity Among Families of Low-SES Backgrounds. Proceedings of the SIGCHI Conference on Human Factors in Computing Systems (CHI 2021). http://doi.org/10.1145/3411764.3445087

[72] Victoria Schwanda, Steven Ibara, Lindsay Reynolds, and Dan Cosley. (2011). Side Effects and "Gateway" Tools: Advocating a Broader Look at Evaluating Persuasive Systems. Proceedings of the SIGCHI Conference on Human Factors in Computing Systems (CHI 2011), 345-348. http://doi.org/10.1145/1978942.1978991

[73] Patrick C. Shih, Kyungsik Han, Erika Shehan Poole, Mary Beth Rosson, and John M. Carroll. (2015). Use and Adoption Challenges of Wearable Activity Trackers. IConference 2015 Proceedings.

[74] Manya Sleeper, William Melicher, Hana Habib, Lujo Bauer, Lorrie Faith Cranor, and Michelle L. Mazurek. (2016). Sharing Personal Content Online: Exploring Channel Choice and Multi-Channel Behaviors. Proceedings of the SIGCHI Conference on Human Factors in Computing Systems (CHI 2016), 101-112. https://doi.org/bwgf

[75] Katarzyna Stawarz, Anna L. Cox, and Ann Blandford. (2015). Beyond Self-tracking and Reminders: Designing Smartphone Apps that Support Habit Formation. Proceedings of the SIGCHI Conference on Human Factors in Computing Systems (CHI 2015), 2653-2662. http://doi.org/10.1145/2702123.2702230

[76] Dawn Surgenor, Lynsey Hollywood, Sinéad Furey, Fiona Lavelle, Laura McGowan, Michelle Spence, Monique Raats, Amanda McCloat, Elaine Mooney, Martin Caraher, and Moira Dean. (2017). The Impact of Video Technology on Learning: A Cooking Skills Experiment. Appetite, 114, 306-312. http://doi.org/10.1016/j.appet.2017.03.037

[77] Lie Ming Tang and Judy Kay. (2017). Harnessing Long Term Physical Activity Data-How Long-term Trackers Use Data and How an Adherence-based Interface Supports New Insights. Proceedings of the ACM on Interactive, Mobile, Wearable and Ubiquitous Technologies (IMWUT 2017), 1-28. http://doi.org/10.1145/3090091

[78] Rannie Teodoro and Mor Naaman. (2013). Fitter with Twitter: Understanding Personal Health and Fitness Activity in Social Media. Proceedings of the International AAAI Conference on Weblogs and Social Media (ICWSM 2013), 611-620.

[79] Rosemary Thackeray, Benjamin T. Crookston, and Joshua H. West. (2013). Correlates of Health-Related Social Media Use Among Adults. Journal of Medical Internet Research 15. http://doi.org/10.2196/jmir.2297

[80] The Clue Encyclopedia: Women's Health, Menstrual Cycle Health, \& Sex. https://helloclue.com/articles

[81] Michela Del Vicario, Alessandro Bessi, Fabiana Zollo, Fabio Petroni, Antonio Scala, Guido Caldarelli, H. Eugene Stanley, and Walter Quattrociocchi. (2016). The Spreading of Misinformation Online. Proceedings of the National Academy of Sciences of the United States of America, 113(3), 554-559. http://doi.org/10.1073/pnas.1517441113

[82] Elizabeth C. Voth, Nelly D. Oelke, and Mary E. Jung. (2016). A Theory-Based Exercise App to Enhance Exercise Adherence: A Pilot Study. FMIR mHealth and uHealth, 4(2), e4997. http://doi.org/10.2196/mhealth.4997

[83] Donald A. Williamson, P. Davis Martin, M.A. White, R. Newton, H. Walden, E. York-Crowe, A. Alfonso, S. 
Gordon, and D. Ryan. (2005). Efficacy of an Internet-Based Behavioral Weight Loss Program for Overweight Adolescent African-American Girls. Eating and Weight Disorders, 10(3), 193-203. https://doi.org/grp9

[84] Zhiying Yue and Michael Stefanone. (2020). Mass Media Consumption and Sexual Image Sharing from a Social Cognitive Perspective. Proceedings of the International Conference on Social Media and Society (SMSociety 2020), 193-199. http://doi.org/10.1145/3400806.3400829

[85] Xuan Zhao, Cliff Lampe, and Nicole B. Ellison. (2016). The Social Media Ecology: User Perceptions, Strategies and Challenges. Proceedings of the SIGCHI Conference on Human Factors in Computing Systems (CHI 2016). http://doi.org/10.1145/2858036.2858333

[86] Yaguang Zheng, Juliet Mancino, Lora E. Burke, and Karen Glanz. (2017). Current Theoretical Bases for Nutrition Intervention and Their Uses. In Nutrition in the Prevention and Treatment of Disease. Elsevier, 185-201. http://doi.org/10.1016/B978-0-12-802928-2.00009-6

Received April 2021; accepted July 2021. 